\title{
Myeloid cell-specific serine palmitoyltransferase subunit 2 haploinsufficiency reduces murine atherosclerosis
}

\author{
Mahua Chakraborty, ${ }^{1}$ Caixia Lou, ${ }^{1,2}$ Chongmin Huan, ${ }^{1}$ Ming-Shang Kuo, ${ }^{3}$ Tae-Sik Park, ${ }^{4}$ \\ Guoqing Cao, ${ }^{3,5}$ and Xian-Cheng Jiang ${ }^{1,6}$ \begin{abstract}
2Institute of Chinese Minority Traditional Medicine, Minzu University of China, Beijing, People's Republic of China. ${ }^{3}$ Lilly Research Laboratories, Eli Lilly and Company, Indianapolis, Indiana, USA. ${ }^{4}$ Department of Medicine, Lee Gil Ya Cancer and Diabetes Institute, Gachon University of Medicine and Science, Incheon, Republic of Korea. 5Jiangsu Hengrui Medicine, Shanghai, People’s Republic of China. 6 Molecular and Cellular Cardiology Program,
\end{abstract} \\ 1Department of Cell Biology, State University of New York (SUNY) Downstate Medical Center, New York, New York, USA. \\ VA New York Harbor Healthcare System, New York, New York, USA
}

\begin{abstract}
Serine palmitoyltransferase (SPT) is the first and rate-limiting enzyme of the de novo biosynthetic pathway of sphingomyelin (SM). Both SPT and SM have been implicated in the pathogenesis of atherosclerosis, the development of which is driven by macrophages; however, the role of SPT in macrophage-mediated atherogenesis is unknown. To address this issue, we have analyzed macrophage inflammatory responses and reverse cholesterol transport, 2 key mediators of atherogenesis, in SPT subunit 2-haploinsufficient (Sptlc2 ${ }^{+/-}$) macrophages. We found that Sptlc2 $2^{+-}$macrophages have significantly lower SM levels in plasma membrane and lipid rafts. This reduction not only impaired inflammatory responses triggered by TLR4 and its downstream NF- $\kappa B$ and MAPK pathways, but also enhanced reverse cholesterol transport mediated by ABC transporters. LDL receptor-deficient $\left(\mathrm{Ldll}^{-/-}\right)$mice transplanted with $S p t l \mathrm{C}^{+/-}$bone marrow cells exhibited significantly fewer atherosclerotic lesions after high-fat and high-cholesterol diet feeding. Additionally, $\mathrm{Ldl}^{-/-}$mice with myeloid cell-specific Sptlc2 haploinsufficiency exhibited significantly less atherosclerosis than controls. These findings suggest that SPT could be a novel therapeutic target in atherosclerosis.
\end{abstract}

\section{Introduction}

Atherosclerosis is a chronic inflammatory disease. The accumulation of macrophage-derived foam cells on the vessel wall is always accompanied by the production of a wide range of chemokines and cytokines (1). These factors regulate the turnover and differentiation of immigrating and resident cells, eventually influencing plaque development. One of the key regulators of inflammation is NF- $\mathrm{NB}(2,3)$, which can be activated by a wide range of signals, including TNF- $\alpha$, IL-1, LPS, and CD40, through different receptors $(4,5)$ on the macrophages.

Foam cell formation due to excessive accumulation of cholesterol by macrophages is another pathological hallmark of atherosclerosis (6). Macrophage scavenger receptor class A is implicated in the deposition of cholesterol on arterial walls during atherogenesis, via receptor-mediated endocytosis of chemically modified LDLs (7). However, macrophages cannot limit the uptake of cholesterol, and therefore depend on cholesterol efflux pathways to prevent their transformation into foam cells. Several ATP-binding cassette (ABC) transporters, including ABCA1 (8) and ABCG1 (9) as well as scavenger receptor class B1 (SR-B1) (9), facilitate the efflux of cholesterol from macrophages. In experiments with isolated macrophages, combined ABCA1 and ABCG1 deficiency resulted in impaired cholesterol efflux to HDL and apoA-I, profoundly decreasing apoE secretion (10). Additionally,

Authorship note: Mahua Chakraborty, Caixia Lou, and Chongmin Huan contributed equally to this work.

Conflict of interest: The authors have declared that no conflict of interest exists. Citation for this article: J Clin Invest. 2013;123(4):1784-1797. doi:10.1172/JCI60415. bone marrow from double-KO $\mathrm{Abca1^{-/- }} A b c g 1^{-/-}$mice transplanted into $\mathrm{Ldlr}^{-/}$mice accelerated atherosclerosis $(10,11)$.

Most recent reports have established that a link exists between macrophage cholesterol efflux and inflammation. In general, more cholesterol efflux is related to less inflammation $(12,13)$. The researchers attributed this phenomenon to altered cholesterol levels in macrophage plasma membrane lipid rafts $(12,13)$. However, it is unknown whether macrophage membrane sphingomyelin (SM) changes affect inflammation and cholesterol efflux, thereby contributing to atherosclerosis.

The interaction of SM, cholesterol, and glycosphingolipid drives the formation of plasma membrane rafts (14). As much as $70 \%$ of all cellular SM is found in these rafts $(15,16)$, and they have been shown to be involved in cell signaling, lipid and protein sorting, and membrane trafficking $(14,17,18)$. Both class A and class B scavenger receptors (19-21), including LDL receptor-related protein (22) and CD36 (23), are located on these SM-rich membrane rafts and/or caveolae. It is also well known that TLRs and inflammatory response mediators are located in the lipid rafts of cells, including macrophages (24-27). It is therefore conceivable that fundamental changes in the SM levels of macrophage plasma membranes modulate macrophage functions in terms of inflammatory responses and cholesterol efflux, thus influencing atherosclerosis development.

Serine palmitoyltransferase (SPT) is the rate-limiting enzyme involved in the biosynthesis of SM (28). Mammalian SPT holoenzyme is primarily a heterodimer, composed of 2 protein subunits - SPTLC1 (53 kDa) and SPTLC2 (63 kDa) - with 20\% sequence homology $(29,30)$. However, recent studies indicate the existence 
A
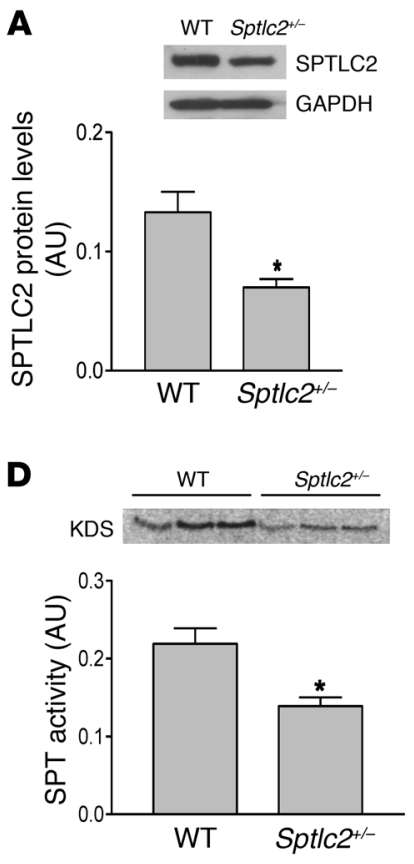

B

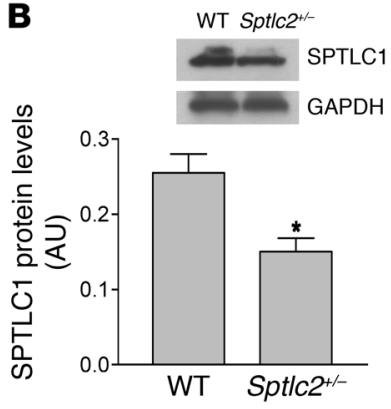

E

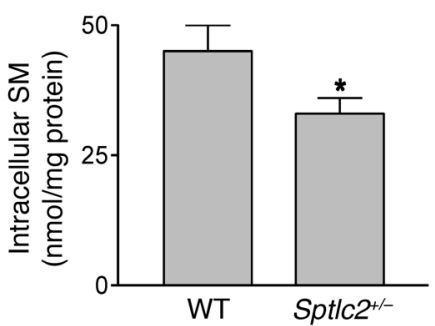

C

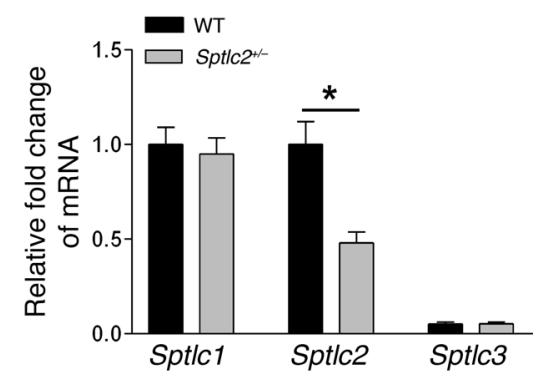

\section{Figure 1}

Characterization of bone marrow-derived Spt/c2+/- macrophages. Bone marrow-derived macrophages were isolated from WT and Sptlc2+/- mice. (A and B) Sptlc2+/- macrophages exhibited reduced expression of SPTLC2 and SPTLC1 subunits, as measured by Western blot from whole cell lysates with densitometric quantification. (C) Total RNA was extracted using TRIzOL method, and Spt/c1, Spt/c2, and Spt/c3 mRNA were measured by real-time PCR using specific primers. (D) SPT activity in Spt/c2+/- macrophages with densitometric quantification. SPT activity in bone marrowderived macrophage homogenate was measured using $\left[{ }^{14} \mathrm{C}\right]$-serine and palmitoyl-CoA as substrates. The product, $\left[{ }^{14} \mathrm{C}\right]-\mathrm{keto}-\mathrm{dihydrosphingosine}$ (KDS), was separated from the reaction mix by thin layer chromatography, and the plate was scanned using Phosphorimager. (E) Intracellular SM content in macrophages was measured by LC/MS/MS. Data are representative of 3 independent experiments $\left(n=4\right.$ per group). ${ }^{*} P<0.05$.

of a third subunit, SPTLC3, with $68 \%$ homology to SPTLC2 (31). Interestingly, the expression of Sptlc3 is almost negligible in hematopoietic tissues, such as PBMCs, macrophages, bone marrow, or spleen. In fact, SPTLC3 levels are compensated by increased expression of Sptlc2 in these tissues (32). Additionally, there are 2 low-molecular weight proteins, ssSPTa and ssSPTb, that enhance enzyme activity and confer distinct acyl-CoA substrate specificities to mammalian SPT, like the yeast Tsc3p subunit (33).

Compelling evidence from multiple studies has established that inhibition of SPT by myriocin results in lower plasma sphingolipid levels in Apoe $e^{-/-}$mice, leading to reduced atherosclerosis (34-36). Mechanisms that link sphingolipid metabolism and atherogenicity include (a) reduction of sphingolipid levels, including SM, ceramide, and sphingosine-1-phosphate (S1P), in the circulation, affecting atherogenicity (35); and (b) reduction of macrophage plasma membrane sphingolipid levels, especially those enriched in lipid rafts, thereby influencing lipid raft-associated cellular functions (inflammation and cholesterol efflux). In the present study, we investigated the relationship between macrophage SPT haploinsufficiency and atherosclerosis. In particular, we focused on macrophage-mediated inflammatory responses and cholesterol efflux. We used bone marrow-derived macrophages from Sptlc2 heterozygous KO (Sptlc2+-) mice (37) as our model system, as homozygous Sptlc2 $2^{-/}$mice are embryonic lethal (37). We showed that Sptlc2 haploinsufficiency in macrophages mitigated inflammatory responses and enhanced cholesterol efflux, which led to attenuation of atherosclerosis in mouse models.

\section{Results}

Sptlc2 haploinsufficiency results in decreased SPT activity, leading to reduced macrophage SM content. Bone marrow-derived Sptlc2 $2^{+-}$ macrophages had 55\% less SPTLC2 protein mass than WT macrophages, as evidenced by Western blot (Figure 1A). Moreover, SPTLC1 mass decreased by about 50\% in Sptlc2 $2^{+/-}$macrophages (Figure 1B), which indicates that SPTLC1 and SPTLC2 should interact with each other, and are not otherwise stable. Moreover, real-time PCR analysis showed that Sptlc2 haploinsufficiency reduced macrophage Sptlc2 mRNA by about 53\% versus WT controls $(P<0.05)$, but had no significant effect on Sptlc1 mRNA levels (Figure 1C). Additionally, there was very low Sptlc3 mRNA in macrophages, owing to its hematopoietic origin. We also measured functional activity of the enzyme and found that SPT activity in Sptlc2 $2^{+-}$macrophages was decreased by $30 \%$ compared with WT controls $(P<0.05$; Figure 1D).

Because it is the first enzyme in the SM biosynthetic pathway, we anticipated that lower SPT activity would lead to a reduction of subsequent lipid metabolites in the pathway, which could play an important role in macrophage function. To test this, we subjected Sptlc2 $2^{+-}$and WT macrophage homogenates to liquid chromatography/mass spectrometry/mass spectrometry (LC/MS/MS) in order to measure the intracellular levels of different sphingolipids. SM levels were reduced by $27 \%$ in Sptlc2 ${ }^{+/-}$versus WT macrophages $(P<0.05$; Figure 1E), whereas ceramide, phosphatidylcholine, diacylglycerol, sphingosine, and S1P showed no significant differences between the groups (Supplemental Table 1; supplemental mate- 


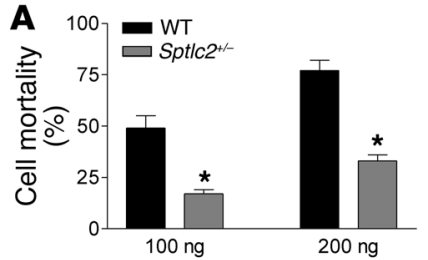

B

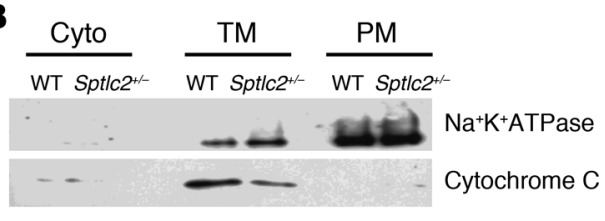

C

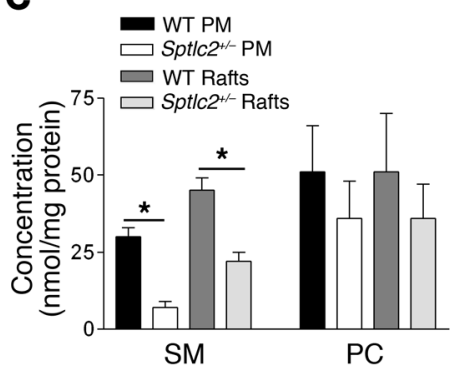

D

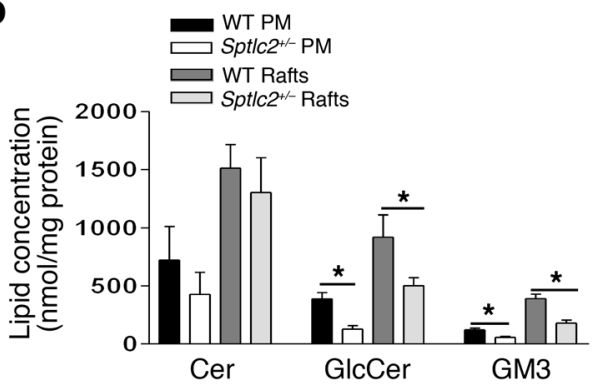

$\mathbf{E}$

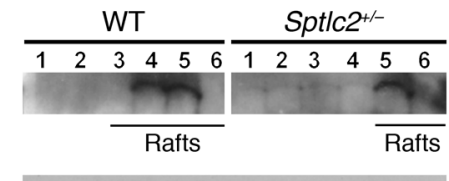

CAV-1

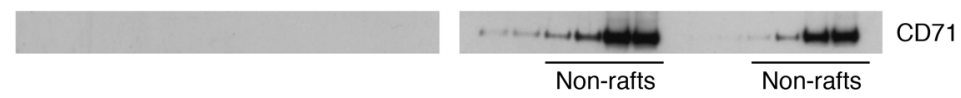

Figure 2

Sptlc2 haploinsufficiency in macrophages leads to significant reductions of SM, glucosylceramide, and GM3 in macrophage plasma membranes and lipid rafts, resulting in altered raft distribution. (A) Cell surface SM measurement by lysenin-mediated cell lysis assay, presented as percent cell mortality. (B) Plasma membrane isolation from primary macrophages. The purity of cytoplasm (Cyto), total subcellular organelle membranes (TM), and plasma membranes (PM) was determined by Western blot; $\mathrm{Na}^{+} / \mathrm{K}^{+}$ATPase was used as a plasma membrane marker, and cytochrome $\mathrm{C}$ as a mitochondrial marker. (C) SM and phosphatidylcholine (PC) measurements in macrophage plasma membranes and lipid rafts using LC/MS/MS. (D) Ceramide (Cer), glucosylceramide (GlcCer), and GM3 in macrophage plasma membranes and lipid rafts, as measured by LC/MS/MS. Plasma membrane measurements were done using equal numbers of macrophages $\left(\sim 50 \times 10^{6}\right.$ cells per group), and lipid raft measurements were performed using equal quantities of proteins from macrophage lysates. (E) Lipid rafts were isolated from macrophages using sucrose density gradient centrifugation, and fractions were collected. Western blots were performed for CAV-1 (raft marker) and CD71 (non-raft marker) with respective antibodies. Data are representative of 3 independent experiments ( $n=6$ per group). ${ }^{*} P<0.05$. rial available online with this article; doi:10.1172/JCI60415DS1). However, the SM changes did not result in any morphological alterations in the Sptlc2 $2^{+-}$macrophages or in their survival in ex vivo culture (data not shown).

Sptlc2 haploinsufficiency results in decreased plasma membrane SM and altered lipid rafts in macrophages. Because a major proportion of cellular SM is located in the membrane rafts $(15,16)$, we investigated how Sptlc2 haploinsufficiency affects plasma membrane and lipid raft SM levels in macrophages. To estimate cell surface SM in Sptlc2 $2^{+-}$macrophages, we performed lysenin assays. Lysenin is a SM-specific cytotoxin that binds to SM-enriched microdomains in plasma membranes, causing lysis of the cells. Therefore, lyseninmediated cell mortality indirectly reflects SM levels on cell surfaces. We found that Sptlc2 ${ }^{+/-}$macrophages showed significantly less sensitivity to lysenin-mediated cytolysis than control cells (Figure 2A), which indicates that Sptlc2 haploinsufficiency decreases SM levels on macrophage cell surfaces.

To confirm this significant change in surface SM of Sptlc2 $2^{+/-}$ macrophages, we next sought to directly measure sphingolipids in plasma membrane. We isolated plasma membranes from the macrophages, the purity of which was checked by Western blot using specific markers. The plasma membrane marker $\mathrm{Na}^{+} /$ $\mathrm{K}^{+}$ATPase was well detected, whereas the mitochondrial marker cytochrome $\mathrm{C}$ could not be traced in the plasma membrane fractions (Figure 2B), indicative of a satisfactory state of purity in the plasma membrane preparations. We then used LC/MS/ MS to quantify plasma membrane sphingolipid levels. Sptlc2+/macrophage plasma membranes displayed significantly less SM, glucosylceramide, and ganglioside (GM3), but did not show significant changes in ceramide or phosphatidylcholine, compared with WT controls (Figure 2, C and D).
Furthermore, we measured SM levels in lipid rafts from the macrophages. For this purpose, cold detergent-treated macrophage lysates were fractionated in sucrose density gradients. The lipid rafts containing fractions were identified by a raft-specific marker, caveolin-1 (CAV-1), while the others were confirmed with a nonraft marker, CD71. Interestingly, in WT macrophages, CAV-1 was equally concentrated in raft fractions 4 and 5, whereas in Sptlc2 $2^{+-}$ macrophages, CAV-1 was mainly localized in raft fraction 5 (Figure $2 \mathrm{E})$, suggestive of a shift in lipid raft arrangement in Sptlc2 ${ }^{+-}$macrophages. We further pooled raft and non-raft fractions (fractions 4-6 and 9-12, respectively) from individual mouse macrophages and measured sphingolipid concentrations using LC/MS/MS. Similar to the pattern observed in $S p t l c 2^{+/-}$plasma membranes, lipid rafts were found to contain significantly less SM, glucosylceramide, and GM3, but did not show significant changes of ceramide or phosphatidylcholine, in Sptlc2 $2^{+/-}$versus WT macrophages (Figure 2, C and D). On the other hand, non-raft fractions showed no change in sphingolipid levels between the 2 groups of macrophages (data not shown). This finding clearly indicated that Sptlc2 haploinsufficiency alters raft arrangement in Sptlc2 ${ }^{+/-}$macrophages, which may alter macrophage functions mediated by raft-associated receptors and transporters.

Sptlc2 haploinsufficiency impairs macrophage inflammatory responses. Macrophages are known to be critical players in inflammation. Activated macrophages induced by bacterial pathogens, stress signals, and free fatty acids have been shown to produce proinflammatory cytokines, which can exacerbate atherosclerosis. TLRs are critical mediators of these signal transductions in macrophages and are also known to be localized in the lipid rafts. To study how Sptlc2 haploinsufficiency affects inflammatory responses in macrophages, we first examined TLR levels on the surface of 
A

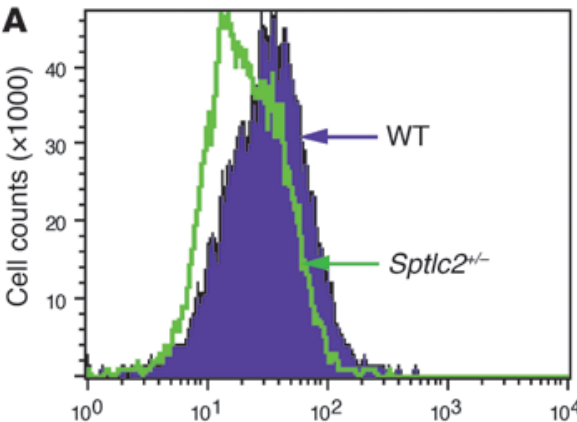

C
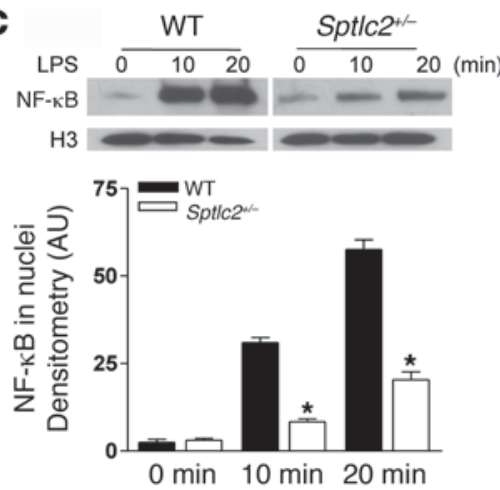

E
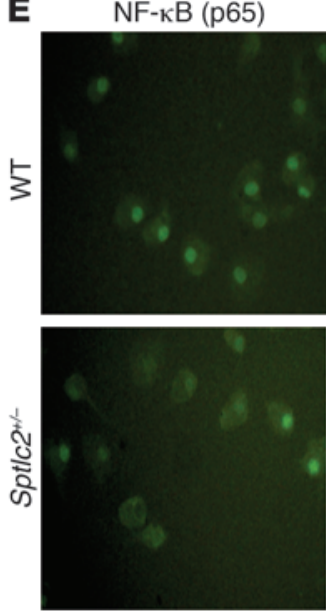

B
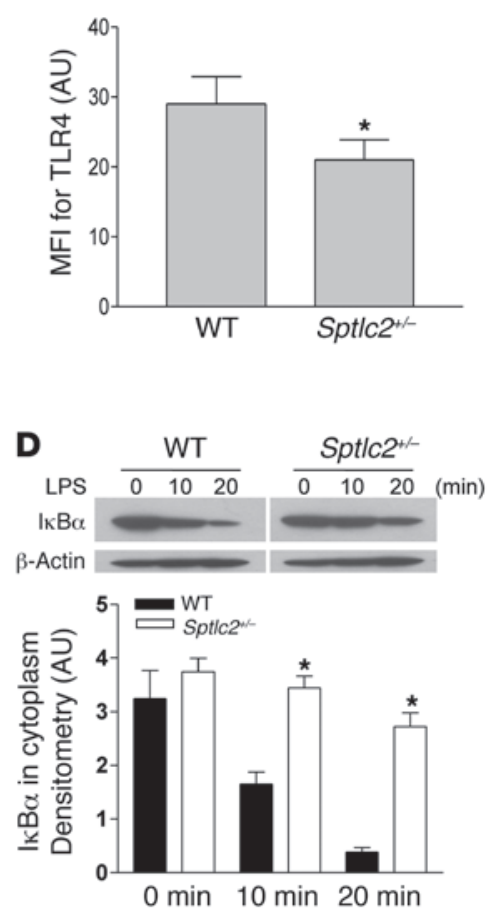

\section{Figure 3}

Spt/C2+/- macrophages attenuate TLR4 recruitment and NF-kB activation after LPS stimulation. (A and B) TLR4 receptors, analyzed by flow cytometry, on the surface of macrophages treated with $10 \mathrm{ng} / \mathrm{ml}$ LPS for 16 hours. (C) Nuclear NF-kB (p65) Western blot and quantitation. (D) Cytosolic IאB $\alpha$ Western blot and quantitation. ( $E$ and $\mathbf{F}$ ) Immunocytochemistry (E) and quantitation (F) of NF-kB (p65) nuclear translocation. Data are representative of 3 independent experiments $(n=3$ per group). ${ }^{*} P<0.05$. macrophages using flow cytometry. Compared with WT controls, Sptlc2 $2^{+-}$macrophages had significantly less TLR4 on their surfaces after stimulation with $10 \mathrm{ng} / \mathrm{ml}$ LPS for 16 hours (Figure 3 , A and B). This indicates that fully functional SPT activity is required to maintain normal TLR levels on macrophage surfaces.

We next sought to determine how Sptlc2 haploinsufficiency affects NF- $\kappa \mathrm{B}$ activation, a key inflammatory signaling pathway down-

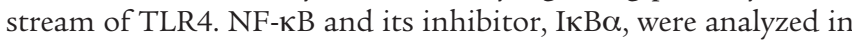
the nuclear and cytoplasmic fractions, respectively, by Western blot after stimulation with $1 \mu \mathrm{g} / \mathrm{ml}$ LPS. Sptlc2 $2^{+/-}$macrophages showed significantly reduced NF- $\mathrm{KB}$ in the nuclear extracts after 10 and 20 minutes of stimulation (61\% and $65 \%$ of WT controls, respectively, $P<0.05$; Figure 3C). Accordingly, the degradation of IкB $\alpha$ in the cytoplasmic extracts of Sptlc2 $2^{+-}$macrophages was markedly slower than that in WT controls (Figure 3D), indicative of attenuated
$\mathrm{NF}-\kappa \mathrm{B}$ activation in Sptlc2 $2^{+/-}$macrophages. To further confirm the blunted NF- $\kappa \mathrm{B}$ activation in Sptlc2 $2^{+/-}$cells, we performed immunocytochemistry for NF- $\mathrm{KB}$ (p65) using macrophages treated with 1 $\mu \mathrm{g} / \mathrm{ml}$ LPS for 15 minutes. Consistent with our Western blot results, Sptlc2 $2^{+/-}$macrophages exhibited reduced nuclear translocation of NF-кB compared with WT controls (Figure 3, E and F).

We also examined activity of MAPKs, another signaling pathway mediated by TLR4 (38), in Sptlc2+/- macrophages, which showed significantly diminished phosphorylation of all 3 MAPKs - ERK, JNK, and p38 - after stimulation with $1 \mu \mathrm{g} / \mathrm{ml}$ LPS compared with WT controls (Figure 4, A-C). Saturated fatty acids, such as palmitate, are also known to induce proinflammatory signaling through TLR4 activation. Sptlc2 $2^{+-}$macrophages also showed significantly reduced MAPK activation when treated with $500 \mu \mathrm{M}$ palmitate (Figure 4, D-F). 
A
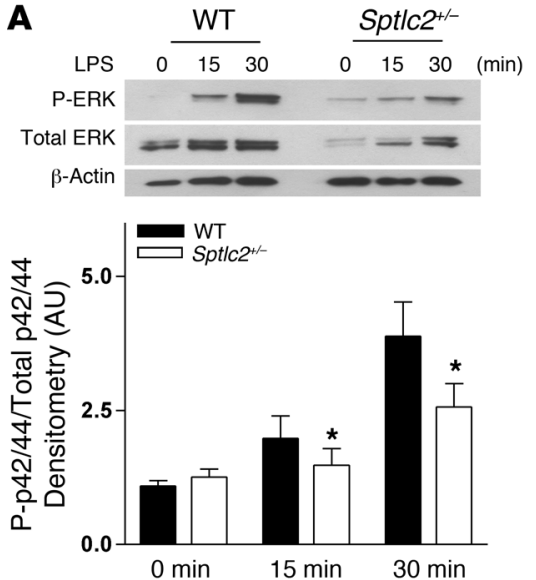

D
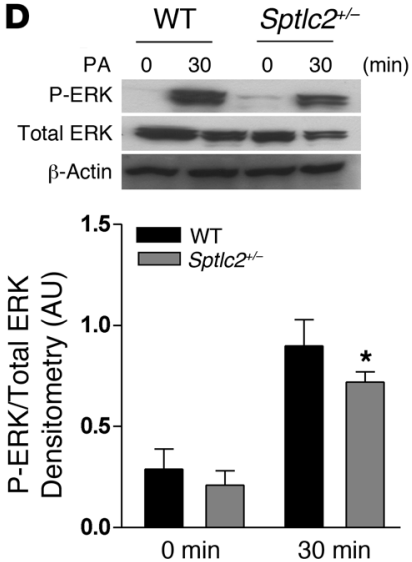

B
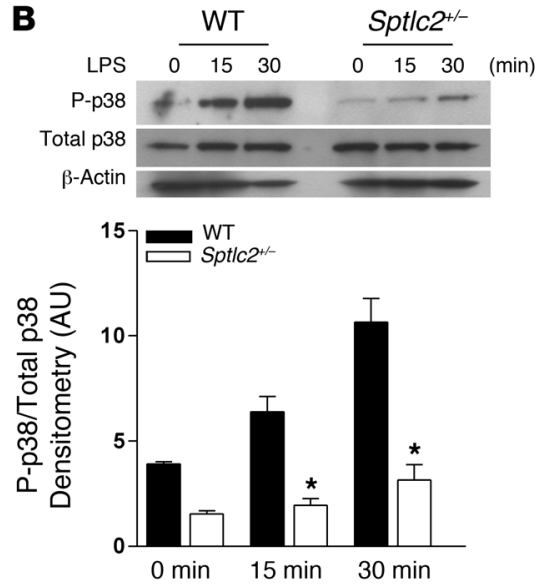

E
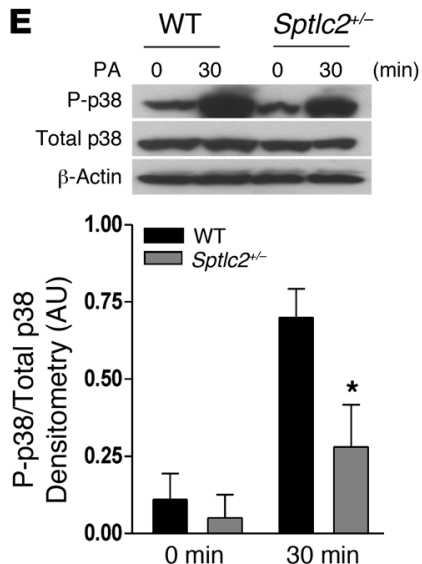
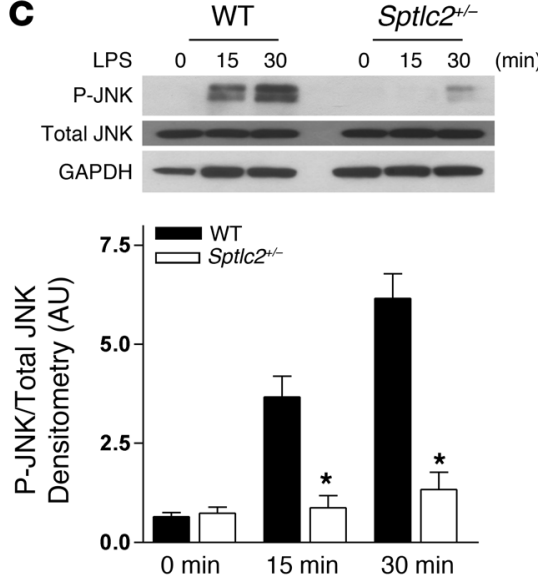

F
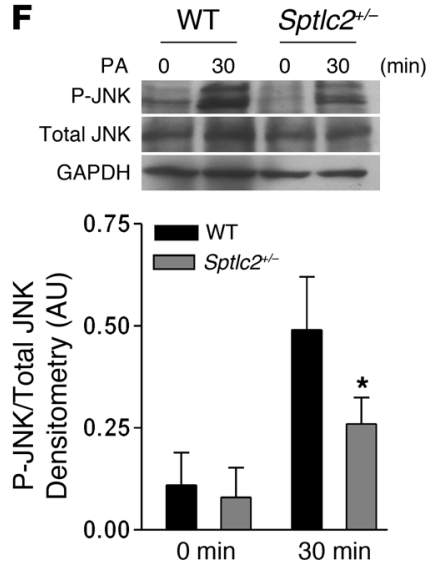

Figure 4

Spt/c2 ${ }^{+-}$macrophages attenuate MAPK activation after LPS or palmitate stimulation. (A and $\left.\mathbf{D}\right)$ ERK, (B and E) p38, and (C and F) JNK (phosphorylated and total protein) Western blot and quantitation after treatment with (A-C) $1 \mu \mathrm{g} / \mathrm{ml}$ LPS or (D-F) $500 \mu \mathrm{M}$ palmitate (PA) in $1 \%$ BSA for 30 minutes (0-minute controls for palmitate were treated with $1 \%$ BSA only). GAPDH and $\beta$-actin were used for loading controls, as indicated. Data are representative of 3 independent experiments $\left(n=3\right.$ per group). ${ }^{*} P<0.05$.

In line with the consequences of attenuated NF-кB and MAPK activation, we observed significantly reduced expression of proinflammatory chemokines and cytokines in Sptlc2 $2^{+/}$macrophages, which produced decreased amounts of MCP-1 in ex vivo cultures (Figure 5A). Moreover, plasma MCP-1 levels were also significantly decreased in Sptlc2 $2^{+/-}$mice at basal level and after i.p. injection of LPS (Figure 5, B and C). To further investigate the consequence of Sptlc2 haploinsufficiency-mediated MCP-1 reduction, we performed an in vitro macrophage migration assay using the Transwell technique. We treated WT and Sptlc2 $2^{+/-}$macrophages with 10 $\mathrm{ng} / \mathrm{ml}$ LPS for 16 hours and collected the culture media. We placed the WT and Sptlc2 $2^{+/-}$macrophage media in the bottom chambers of Transwell plates, added WT and Sptlc2 ${ }^{+/-}$macrophages on the respective top chambers, and monitored macrophage migration. After 4 hours of migration, Sptlc2 $2^{+-}$macrophage medium (with lesser MCP-1 levels) exhibited 34\% reduced chemotactic activity of Sptlc2 $2^{+-}$macrophages compared with that of WT cells in response to WT macrophage medium ( $P<0.05$; Figure 5 , D and E).

Furthermore, Sptlc2 $2^{+-}$macrophages exhibited significantly reduced Tnfa and $I l 6$ mRNA levels compared with WT controls after treatment with $1 \mu \mathrm{g} / \mathrm{ml}$ LPS for 15 minutes or $500 \mu \mathrm{M}$ pal- mitic acid for 30 minutes (Figure 6, A and B). Consistently, Sptlc2 $2^{+-}$ macrophages secreted significantly less TNF- $\alpha$ into culture medium than did WT macrophages after treatment with $10 \mathrm{ng} / \mathrm{ml}$ LPS or $50 \mu \mathrm{M}$ palmitate for 16 hours (Figure 6C). In accordance with the results derived from cultured macrophages, we also found that compared with WT controls, Sptlc2 $2^{+/-}$mice challenged with LPS $(50 \mu \mathrm{g} / \mathrm{kg}$ body weight i.p.) had significantly lower plasma TNF- $\alpha$ and IL-6 levels (Figure 6D). These results demonstrated that Sptlc2 haploinsufficiency impaired the macrophage inflammatory response, which correlated with reduced SM levels in lipid rafts.

To investigate the effect of membrane SM directly, we sought to determine whether exogenous SM supplementation to Sptlc2 $2^{+-}$ macrophages could recapitulate the inflammatory properties of WT macrophages. We used the lysenin assay to evaluate cell membrane SM levels, as described above. SM supplementation enriched the plasma membrane with increased SM, resulting in more lysenin binding and hence more cell mortality. Exogenous supplementation of SM in culture restored the sensitivity of Sptlc2 $2^{+-}$macrophages in response to lysenin treatment (Figure 6E), indicative of recovered SM level in plasma membranes. More importantly, SM supplementation up to $20 \mathrm{nM}$ restored inflammatory func- 

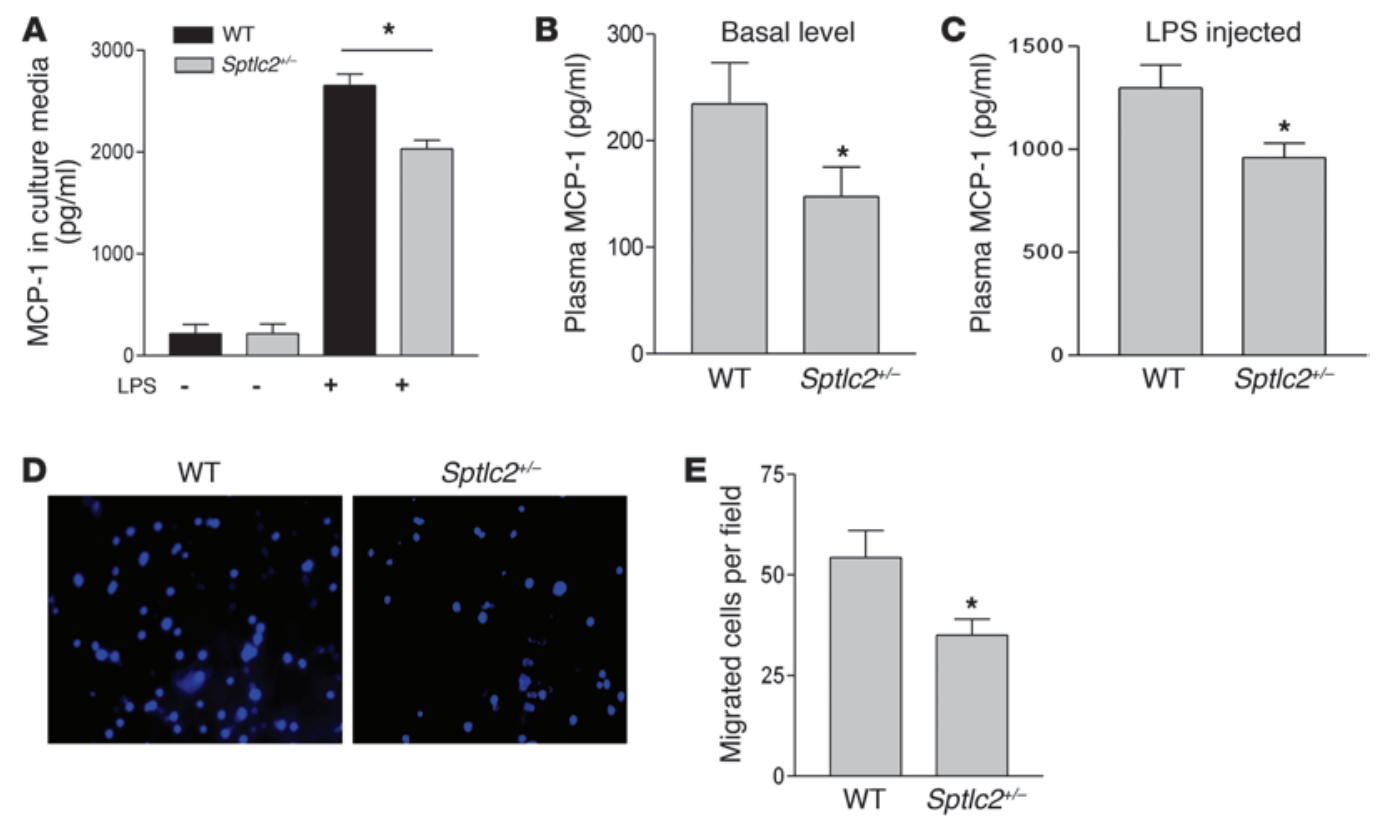

\section{Figure 5}

Spt/c2+/- macrophages exhibit reduced migration under inflammatory conditions. (A) MCP-1 secreted by macrophages in culture medium after treatment with $10 \mathrm{ng} / \mathrm{ml}$ LPS for 16 hours. (B and C) Mouse plasma MCP-1 levels (B) under basal conditions and (C) after 8 hours of LPS challenge $(50 \mu \mathrm{g} / \mathrm{kg}$ body weight i.p.). (D) In vitro Transwell migration of WT and Spt/c2+/- macrophages in response to LPS-treated macrophage culture medium, as seen by DAPI staining. (E) Quantitation of migrated cells in an average of 10 random microscopic fields. Data are representative of 3 independent experiments ( $n=3$ per group). For in vivo studies, $n=7$. ${ }^{*} P<0.05$.

tion in Sptlc2 $2^{+-}$macrophages, as indicated by full recovery of the cells' IL-6 production in response to stimulation with $10 \mathrm{ng} / \mathrm{ml}$ LPS for 16 hours (Figure 6F).

We noted above that in addition to SM, glucosylceramide and GM3 were also reduced in Sptlc2 ${ }^{+/-}$macrophage plasma membranes and lipid rafts (Figure 2, C and D). To rule out their possible contributions in the observed phenotype, we supplemented Sptlc2 ${ }^{+-}$macrophages with different concentrations of glucosylceramide and GM3, followed by LPS treatment and IL- 6 measurement in culture media. However, neither of them had any effect on IL- 6 production by WT or Sptlc2 ${ }^{+/-}$macrophages (Supplemental Figure 1, A and B). Thus, our data strongly supported the notion that reduced membrane SM levels resulting from Sptlc2 haploinsufficiency caused the blunted inflammatory response of the macrophages.

Sptlc2 haploinsufficiency induces cholesterol efflux in macrophages and promotes macrophage-mediated reverse cholesterol transport in mice. Macrophage cholesterol efflux plays an important role in reverse cholesterol transport, an antiatherogenic process. The efflux of cholesterol from macrophages is facilitated by the membrane transport proteins ABCA1, ABCG1, and SR-B1. We found that Sptlc2 haploinsufficiency significantly increased protein levels of ABCA1 (2.2-fold; $P<0.05$ ), but not ABCG1 or SR-B1, in acetylated-LDL-treated macrophages compared with WT controls (Figure 7A). Interestingly, flow cytometry analysis showed that acetylated-LDL-treated Sptlc2+- macrophages exhibited substantially more cell surface ABCA1 and ABCG1 than WT macrophages, as evidenced by shifts in MFI peaks, whereas SR-B1 levels demonstrated no change (Figure 7B). Accordingly, we found that $\left[{ }^{3} \mathrm{H}\right]$-cholesterol efflux mediated by apoA-I or HDL was significantly increased in $S p t l c 2^{+/-}$versus WT macrophages at both 4 and 8 hours (Figure 7, C and D).
This indicates that increased cholesterol efflux in Sptlc2 $2^{+/-}$macrophages was mediated by elevated $\mathrm{ABCA} 1$ and ABCG1 on the surface of these cells, favored by reduced plasma membrane SM.

We next used an approach reported by Rader's group (39) to evaluate the effect of Sptlc2 haploinsufficiency on macrophage cholesterol efflux in vivo. Bone marrow-derived macrophages from WT and Sptlc2 $2^{+/-}$mice were first loaded with $\left[{ }^{3} \mathrm{H}\right]$-cholesterol by incubation with acetylated-LDL, then injected i.p. into C57BL/6 acceptor animals. We found that $S p t l c 2^{+/-}$macrophages significantly increased $\left[{ }^{3} \mathrm{H}\right]$-cholesterol efflux into the circulation compared with WT controls at 6, 24, and 48 hours (3.8-, 2.2-, and 2.7-fold increase, respectively; $n=7 ; P<0.01$; Figure 7E). Furthermore, feces from $S p t l c 2^{+/-}$mice also accumulated more $\left[{ }^{3} \mathrm{H}\right]$-cholesterol compared with WT controls at 24 and 48 hours after initial injection (3.2- and 4.2-fold increase, respectively; $n=7 ; P<0.001$; Figure 7F). These results demonstrated that Sptlc2 haploinsufficiency in macrophage promotes reverse cholesterol transport in vivo, correlated with increased cell surface ABCA1 and ABCG1.

Sptlc2 haploinsufficiency in hematopoietically derived cells reduces atherosclerosis in Ldl/ ${ }^{\prime-}$ mice. To investigate the effects of macrophage Sptlc2 haploinsufficiency on atherosclerosis development, we transplanted Sptlc2 $2^{+/-}$mouse bone marrow into $\mathrm{Ldlr}^{-/}$mice, generating bone marrow chimeric mice referred to herein as Sptlc2 $2^{+-} \rightarrow$ Ldlr $^{-/}$(Supplemental Figure 2A). 8 weeks after transplantation, complete replacement of the bone marrow-derived cells was evidenced by genomic PCR using PBMCs. In the $S p t l c 2^{+/-} \rightarrow L d l r^{-/}$group, PBMCs had been replaced by donor cells of a Sptlc2 $2^{+/-}$genotype, whereas in the WT $\rightarrow L d l r^{-/}$group, the replaced PBMCs were of a WT genotype (Supplemental Figure $2 \mathrm{~B}$ ). The animals were switched to a Western-type diet and periodically checked for body weight gain; gradual weight gain 
A

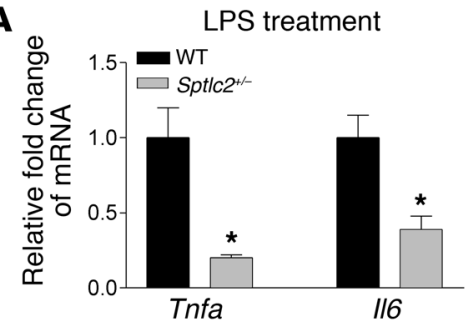

C

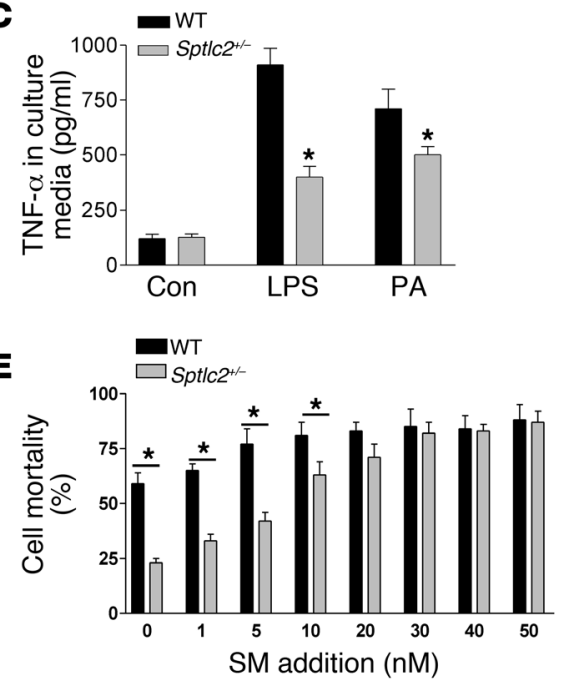

B

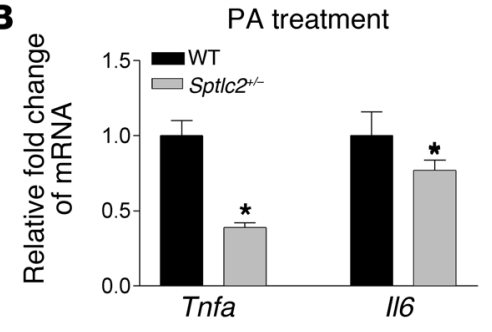

D

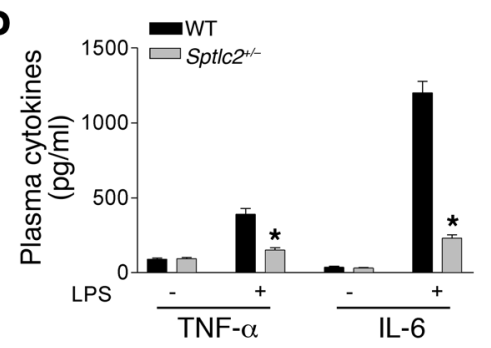

$\mathbf{F}$

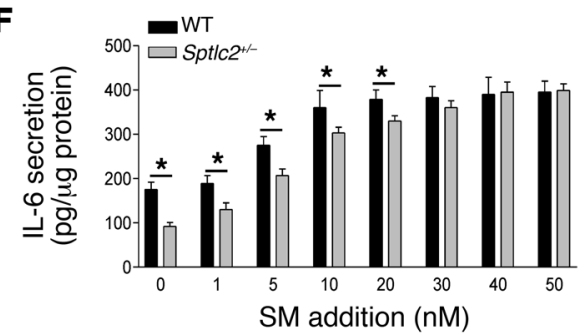

\section{Figure 6}

Spt/c2 $2^{+/}$macrophages attenuate LPS- or palmitate-mediated expression of proinflammatory genes downstream of NF- $\mathrm{KB}$ and MAPKs. (A and B) Real-time PCR for Tnfa and $/ / 6$ mRNA after treatment with (A) $1 \mu \mathrm{g} /$ ml LPS for 15 minutes or (B) $500 \mu \mathrm{M}$ palmitate for 30 minutes. (C) TNF- $\alpha$ secreted by macrophages in culture medium after treatment with $10 \mathrm{ng} / \mathrm{ml}$ LPS or $50 \mu \mathrm{M}$ palmitate for 16 hours. (D) Mouse plasma TNF- $\alpha$ and IL-6 concentration after 8 hours of LPS challenge (50 $\mu \mathrm{g} / \mathrm{kg}$ body weight i.p.). (E) Effect of exogenous SM on lysenin-mediated cell mortality. (F) Effect of exogenous SM on macrophage IL-6 secretion in response to treatment with $10 \mathrm{ng} / \mathrm{ml}$ LPS for 16 hours. Extracellular culture medium IL-6 was measured by ELISA. Data are representative of 3 independent experiments $(n=3)$. For in vivo studies, $n=7 .{ }^{*} P<0.05$. was observed in all animals, but no significant differences were found between groups (Supplemental Figure 3). We also used enzymatic assays to measure fasting plasma cholesterol, triglyceride, phospholipids, and SM at the end of the third month of Western-type diet feeding, but found no significant changes (Supplemental Table 2). Sphingolipid measurement from the plasma samples using LC/MS/MS also indicated no change in SM, ceramide, sphingosine, or S1P levels between groups (Supplemental Table 3). Additionally, we subjected the pooled plasma $(n=9)$ to fast protein liquid chromatography (FPLC) in order to check the lipid distribution, but found no changes (Supplemental Figure 4).

To evaluate whether Sptlc2 haploinsufficiency alters blood cell counts, we analyzed whole blood samples from $S p t l c 2^{+/-}$and WT mice as well as from Sptlc2 $2^{+-} \rightarrow L d l r^{-/}$and WT $\rightarrow L d l r^{-/}$bone marrow chimeric mice; no differences in blood cell counts were observed between the compared groups (Supplemental Tables 4 and 5). Moreover, flow cytometry analysis confirmed that there were no differences in relative percentages of B cells, $\mathrm{T}$ cells, and monocytes/macrophages in the circulation of Sptlc2 $2^{+/-} \rightarrow \mathrm{Ldlr}^{-/}$versus WT $\rightarrow \mathrm{Ldlr}^{-/}$mice (Supplemental Figure 5, A and B). We also performed ELISA in chimeric mice to quantify plasma cytokines and chemokines; Sptlc2 ${ }^{+/-} \rightarrow \mathrm{Ldlr}^{/-}$mice had 55\% less IL-6 $(P<0.01)$ and $47 \%$ less MCP-1 $(P<0.05)$ than that of WT $\rightarrow \mathrm{Ldlr}^{-/}$controls (Supplemental Figure 6, A and B), confirming reduced inflammation in Sptlc2 $2^{+-} \rightarrow L_{d l r^{-1}}$ mice.

After 3 months on a Western-type diet, we dissected mouse proximal aortae and aortic arches, then photographed and measured lesion areas in order to evaluate the effect of macrophage Sptlc2 haploinsufficiency on atherogenesis. We found that all mice (18 of 18) had lesions in the aortic arches; however, those of Sptlc2 ${ }^{+/-} \rightarrow \mathrm{Ldlr}^{-/}$mice were noticeably smaller than those of $\mathrm{WT} \rightarrow \mathrm{Ldll}^{-/}$mice (Figure 8A). In addition, significantly reduced lesion areas were revealed by Oil-Red $O$ staining in the whole aortae of $\mathrm{Sptlc2}^{+/-} \rightarrow \mathrm{Ldll}^{-/}$animals (47\% reduction, $P<0.05$; Figure 8, B and C). The proximal aortae of Sptlc2+/- $\rightarrow L d l r^{-/}$animals also exhibited decreased lesion areas compared with WT $\rightarrow L_{d l r^{-1}}$ specimens ( $34 \%$ decrease, $P<0.05$; Figure $8, \mathrm{D}$ and $\mathrm{E}$ ). To evaluate lesion composition, the aortic root sections were immunostained to determine macrophage accumulation. We found that all sections stained positively for macrophage markers (MOMA), but Sptlc2 $2^{+/} \rightarrow$ Ldlr $^{-/-}$mice had a significant reduction in macrophage content compared with $\mathrm{WT} \rightarrow \mathrm{Ldlr}^{-/}$mice (37\% reduction, $P<0.05$; Figure 8, F and G).

This was caused by reduced MCP-1 production by Sptlc2 ${ }^{+/-}$ macrophages (Supplemental Figure 6B), which led to decreased migration of monocytes and macrophages into the intima of the lesions. Conversely, collagen staining by Masson trichrome and smooth muscle cell actin staining by immunohistochemistry showed no significant differences between the groups (Supplemental Figure 7, A and B).

It has previously been reported that macrophage $\mathrm{S} 1 \mathrm{P}$ receptor (S1PR) activity has an effect on atherogenicity (40). To evaluate whether this is also the case in Sptlc2 haploinsufficiency, we measured S1pr1 and S1pr2 mRNA levels in Sptlc2+-- and WT macrophages by real-time PCR; no significant changes were observed (Supplemental Figure 8, A and B). These results indicate that macrophage Sptlc2 haploinsufficiency inhibits atherogenesis in mice through SM reduction in plasma membranes.

Sptlc2 haploinsufficiency in myeloid cell lineage reduces atherosclerosis in Ldlr/- mice. To obtain more direct evidence to support attribution of the phenotype to Sptlc2 $2^{+-}$macrophages, we crossed our previous- 
A

B
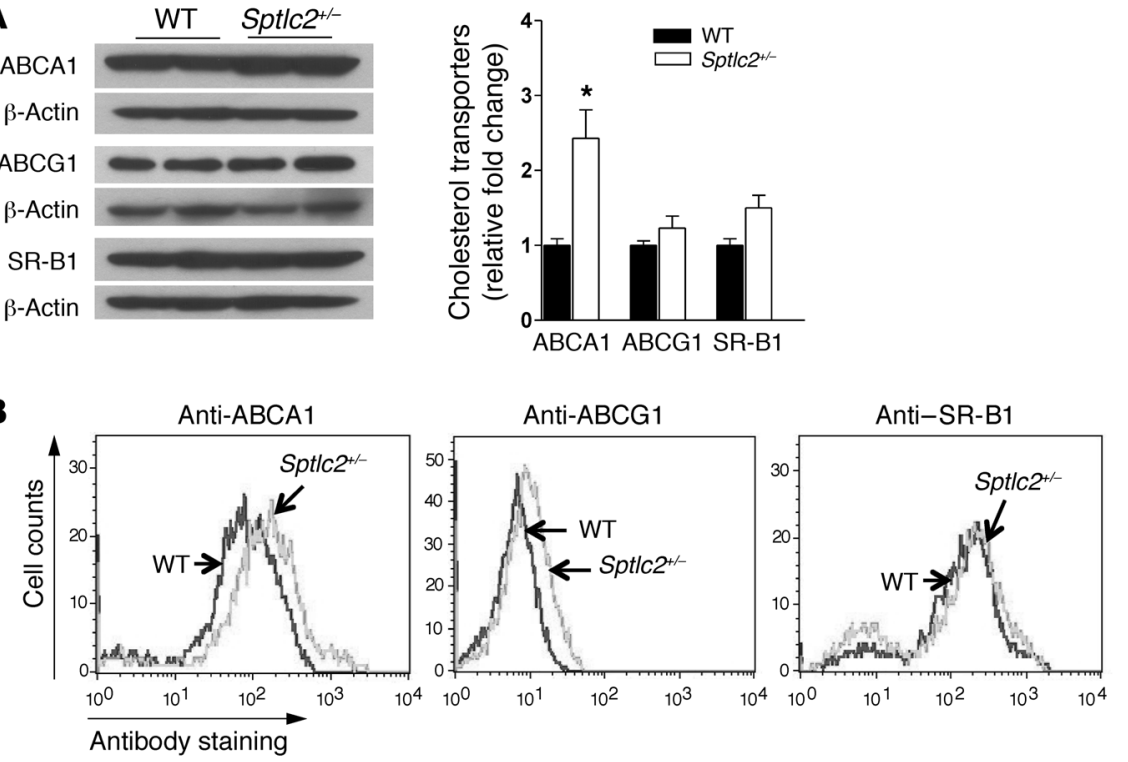
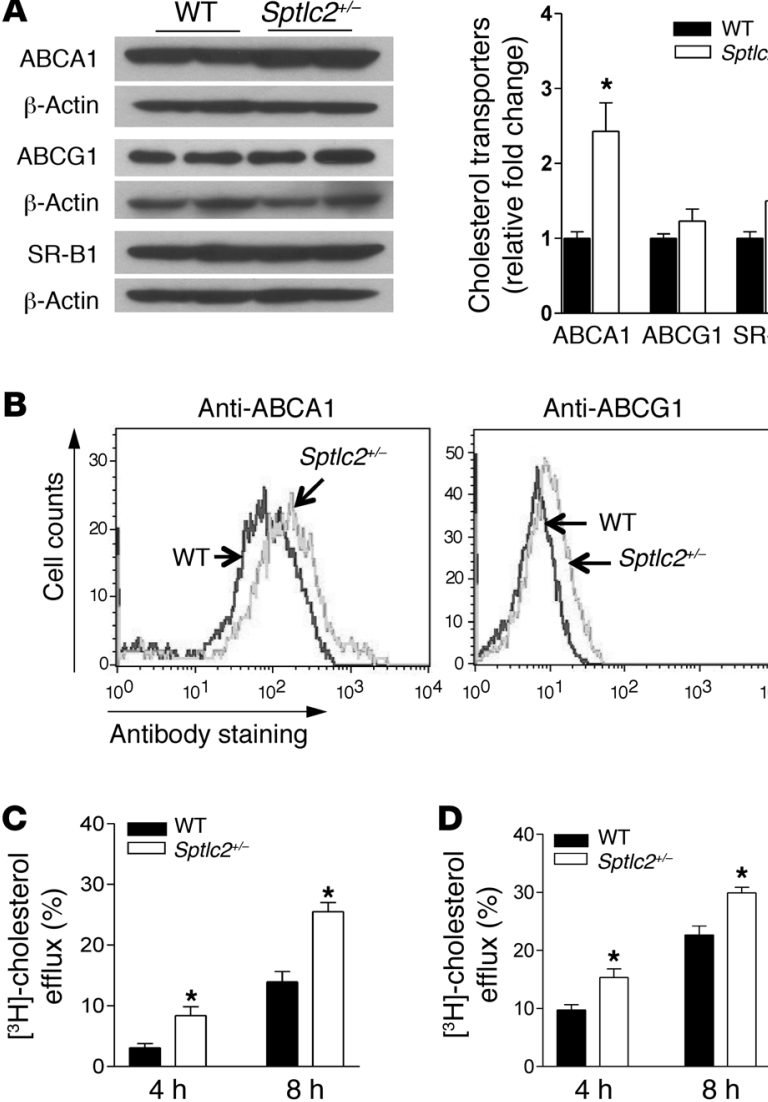

E

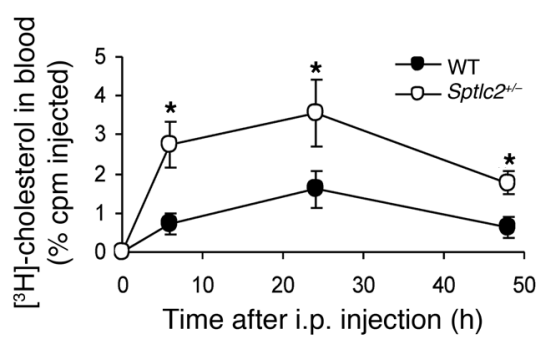

$\mathbf{F}$

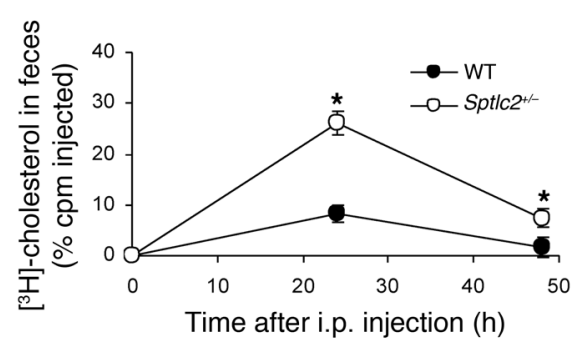

Figure 7

Sptlc2 haploinsufficiency increases macrophage cholesterol efflux, ex vivo and in vivo. (A) Macrophage ABCA1, ABCG1, and SR-B1 Western blot and quantitation after treatment with $50 \mu \mathrm{g} / \mathrm{ml}$ acetylated-LDL for 16 hours. (B) Macrophage surface expression of ABCA1, ABCG1, and SR-B1 after treatment with $50 \mu \mathrm{g} / \mathrm{ml}$ acetylated-LDL for 16 hours, as measured by flow cytometry. (C and $\mathbf{D}$ ) In vitro cholesterol efflux mediated by apoA-I (C) and HDL (D) in WT and Spt/c2 ${ }^{+/-}$macrophages. (E and F) Spt/c2+/- or WT bone marrow-derived macrophages were loaded with cholesterol by incubation with acetylated-LDL and $\left[{ }^{3} \mathrm{H}\right]$-cholesterol. Labeled macrophages were injected i.p. into C57BL/6 acceptor mice. (E) Plasma samples were collected at 6, 24, and 48 hours, and $(\mathbf{F})$ feces were collected at 24 and 48 hours; all were analyzed for tracer counts using liquid scintillation counter. Data are representative of 3 independent experiments ( $n=3$ per group). For in vivo studies, $n=7 .{ }^{*} P<0.01$. ly described Sptlc2-Flox mice (41) with LysM-Cre transgenic mice and prepared heterozygous Sptlc2-Flox/LysM-Cre transgenic mice (Supplemental Figure 9A). The LysM-Cre-mediated recombination results in deletion of the targeted gene in the myeloid cell lineage, including monocytes, mature macrophages, and granulocytes (42). We then transplanted bone marrow cells from Sptlc2-Flox/LysMCre mice into $L d l^{-/-}$mice to obtain myeloid cell-specific Sptlc2haploinsufficient $\mathrm{Ldlr}^{-1-}$ mice (referred to herein as Sptlc2-Flox/ LysM-Cre $\rightarrow L d l^{-/-}$mice; Supplemental Figure 9B). As a control, we transplanted bone marrow cells from Sptlc2-Flox mice into $\mathrm{Ldll}^{-1}$ mice (Sptlc2-Flox $\left.\rightarrow L d l r^{-/}\right)$. Blood cell counts showed no differences between Sptlc2-Flox/LysM-Cre $\rightarrow L d l r^{-/-}$and Sptlc2-Flox $\rightarrow L d l r^{-1-}$ mice (Supplemental Table 6). After 3 months on a Western-type diet, all mice (18 of 18) had lesions in the aortic arches; however, those of Sptlc2-Flox/LysM-Cre $\rightarrow L d l r^{-/-}$mice were smaller than those of Sptlc2-Flox $\rightarrow L_{d l} r^{-}$mice (Figure 9A). The proximal aortae of Sptlc2-Flox/LysM-Cre $\rightarrow L_{d l r^{\prime-}}$ animals also exhibited decreased lesion areas ( $35 \%$ decrease, $P<0.05$; Figure $9, \mathrm{~B}$ and $\mathrm{C})$. In addition, significantly reduced lesion areas were revealed by Oil-Red O staining in the whole aortae of Sptlc2-Flox/LysM-Cre $\rightarrow \mathrm{Ldlr}^{-/}$- compared with control mice ( $30 \%$ reduction, $P<0.05$; Figure 9, D and E).

\section{Discussion}

In this study, we demonstrated a novel and essential role of SPT in modulating macrophage inflammatory responses and cholesterol efflux, thereby influencing atherosclerosis development. Our conclusion is based on the observations that: (a) in Sptlc2 $2^{+/-}$macrophages, LPS-induced membrane recruitment of TLR4-MD2 complex was impaired; (b) ligand-induced (i.e., LPS or palmitate) NF-KB and MAPK activation, as well as proinflammatory cytokine production, were significantly attenuated; and (c) ABCA1- and ABCG1-mediated in vitro and in vivo cholesterol efflux were significantly increased. Additionally, Sptlc2 haploinsufficiency in bone marrow-derived cells or in the myeloid cell lineage led to a decrease in atherosclerosis in $L d l^{-/-}$mice fed a Western-type diet for 3 months. We attribute 

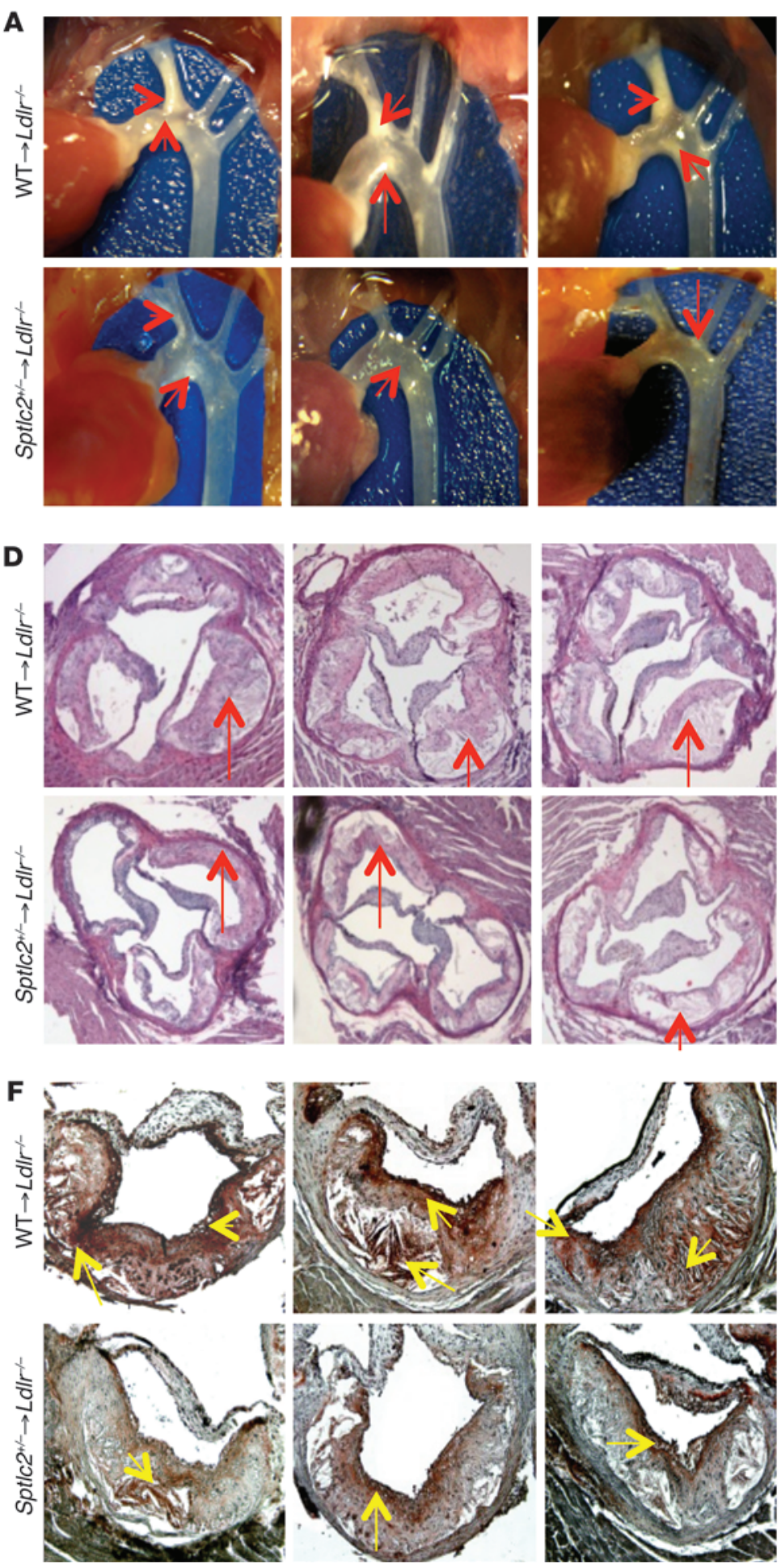

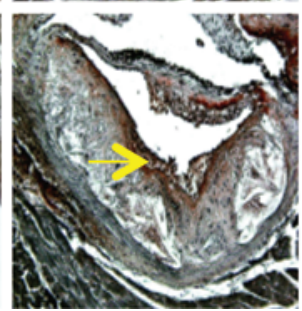

B

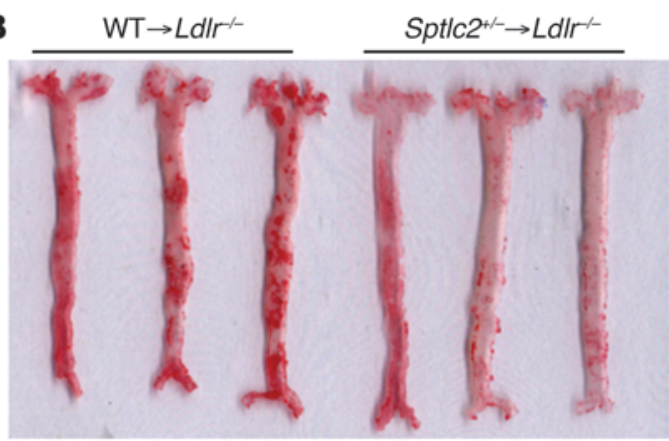

C

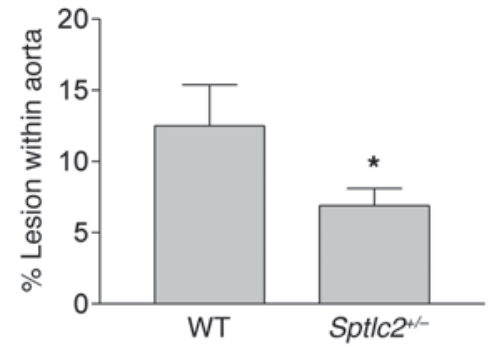

E

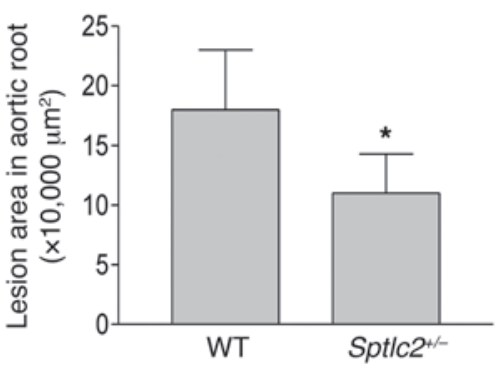

G

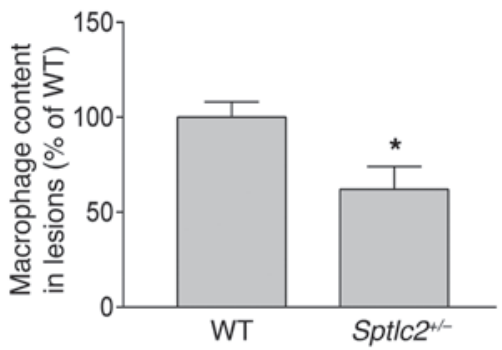

Figure 8

Reduced atherosclerotic lesions in Sptlc2 $2^{+-} \rightarrow$ LdII $r^{-1}$ mice. (A) Aortic arches with atherosclerotic plaques (red arrows). (B) En face aortic plaque analysis after Oil Red O staining. (C) Quantitation of en face assay. (D) Aortic root assay for lesion areas after H\&E staining. Red arrows denote atherosclerotic plaques. (E) Quantitation of root assay. (F) Immunohistochemical staining of macrophage accumulation in lesions (brown-stained regions, denoted by yellow arrows). (G) Quantitation of macrophage content. Quantitative analyses were done using ImageJ; 6 alternate sections (10 $\mu \mathrm{m}$ thick) sliced from paraffin-fixed aortic root tissues of each transplanted mouse were used for analysis. $n=9 .{ }^{*} P<0.05$. 

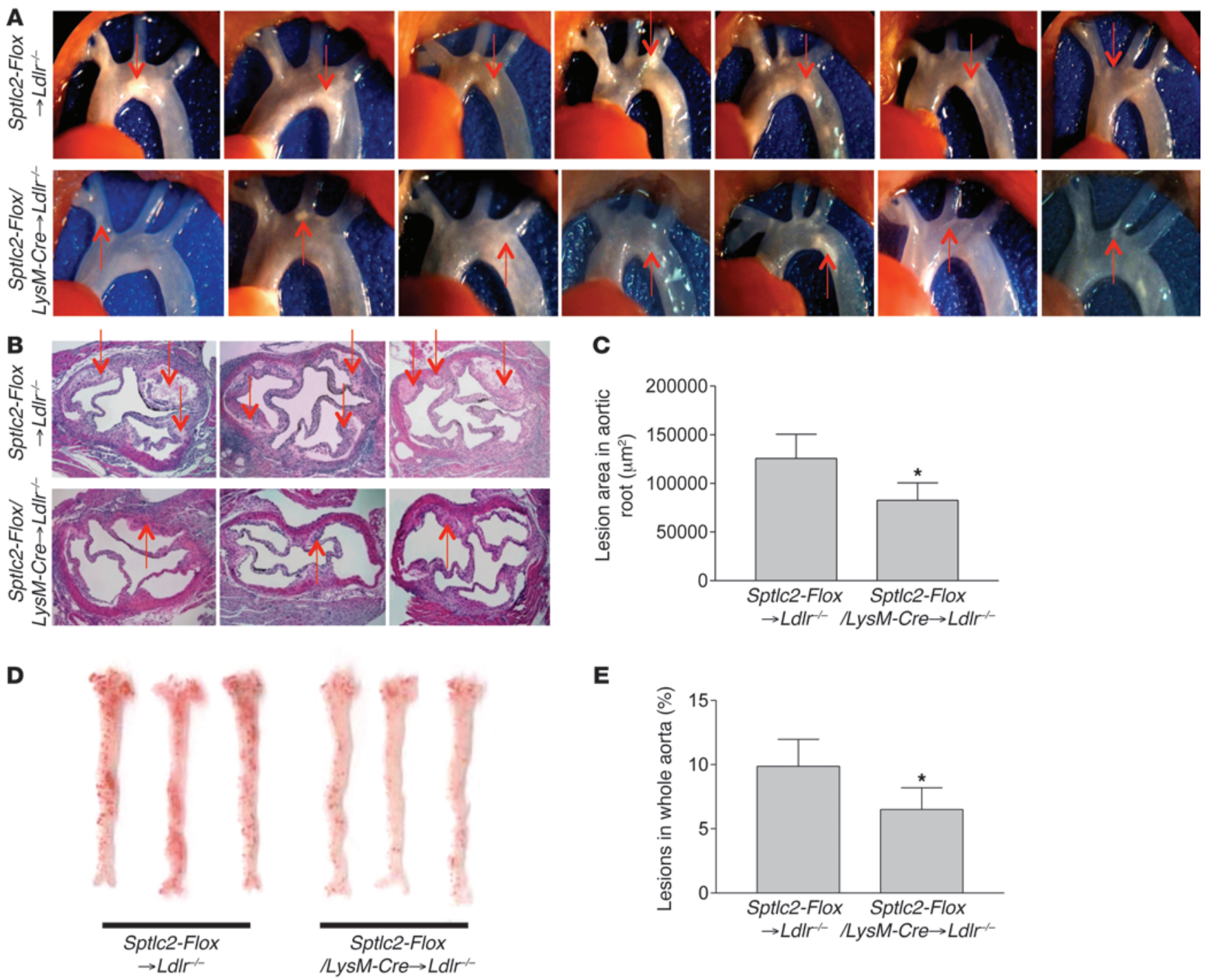

$\mathbf{E}$

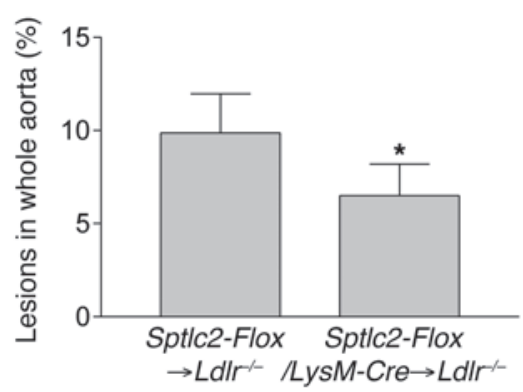

Figure 9

Reduced atherosclerotic lesions in Spt/c2-Flox/LysM-Cre $\rightarrow$ Ldll $r^{-1}$ mice. (A) Aortic arches with atherosclerotic plaques (red arrows). (B) Aortic root assay for lesion areas after H\&E staining. 6 alternate sections (10 $\mu \mathrm{m}$ thick) sliced from paraffin-fixed aortic root tissues of each transplanted mouse were used for analysis. Red arrows denote atherosclerotic plaques. (C) Quantitation of root assay. (D) En face aortic plaque analysis after Oil Red O staining. (E) Quantitation of en face assay. $n=9$. ${ }^{*} P<0.05$.

this SPT haploinsufficiency-mediated antiatherogenic property to SM reduction in the macrophage plasma membranes.

Previous studies have indicated that treatment with myriocin, a potent SPT inhibitor, ameliorates atherosclerosis in $\mathrm{Apoe}^{-/-}$ mice $(34,35)$. In addition, we and others previously observed that myriocin treatment decreases all sphingolipids, including SM, ceramide, and glycosphingolipids (34-36), in all tissues tested, including macrophages. However, it was not known which sphingolipids in particular (from the de novo sphingolipid biosynthetic pathway) were responsible for reducing atherosclerosis. Our present findings indicate that cellular SM is one of the major factors modulating macrophage atherogenic functions, as levels of SM - but not ceramide or phosphatidylcholine - in cell homogenates, plasma membranes, and membrane lipid rafts were significantly decreased in Sptlc2 ${ }^{+/-}$macrophages (Figure 1E, Figure 2, C and D, and Supplemental Table 1).
A recent report indicated that decreased SM levels in plasma membrane lipid rafts can diminish the development of obesity and type 2 diabetes (43), which are closely related to atherosclerosis. We observed similar phenomena in our Sptlc2 $2^{+/-}$mice and in SM synthase $2-\mathrm{KO}\left(\right.$ Sms $\left.2^{-{ }^{-}}\right)$mice (44). Thus, it is conceivable that Sptlc2 insufficiency-mediated membrane SM reduction could have consequences in terms of macrophage inflammation and cholesterol efflux, the critical factors in atherogenesis.

Plasma membrane SM reduction could diminish signal transduction mediated by lipid raft-associated receptors, such as TLR4. As reported previously, interaction of SM and cholesterol drives plasma membrane raft formation (14), and the relative proportions of both SM and cholesterol appear to be critical for the stability and function of lipid rafts $(14,45)$. In the present study, we found that LPS-induced plasma membrane recruitment of the TLR4-MD2 complex was diminished in Sptlc2+/- macrophages 
(Figure 3, A and B), thereby reducing both NF-кB and MAPK activation and attenuating inflammatory cytokine production. To further confirm our conclusion that SM directly affects inflammatory signaling, we performed SM add-back experiments, which demonstrated that exogenous SM could enrich plasma membrane SM levels and eventually restore the WT inflammatory phenotype in Sptlc2 ${ }^{+/-}$macrophages (Figure 6, E and F).

$\mathrm{SM}$ is also known as a cholesterol-binding molecule and plays an important role in cholesterol efflux. This can be explained by 2 possible mechanisms. First, SM is involved in the recruitment of effluxrelated transporters to the plasma membrane (46). It is therefore conceivable that plasma membrane SM changes in macrophages could influence the functions of these proteins, altering cholesterol efflux. Indeed, enhanced apoA-I-dependent cholesterol efflux by ABCA1 from SM-deficient cells has been reported previously (47). Here, we found that decreased SM levels in macrophage plasma membrane increased protein levels of both ABCA1 and ABCG1 on macrophage surfaces, thereby increasing cholesterol efflux in vitro and in vivo (Figure 7, B-F). Although ABCA1 is known to be located in a non-raft region, its levels influence lipid raft structure (48). It has been reported that overexpression of ABCA1 (49) and treatment of cells with HDL or apoA-I $(50,51)$ disrupts or depletes raft domains, inhibiting raft-dependent signaling. Thus, there is an interaction between ABCA1 and raft lipids. Moreover, SM is also critical for cholesterol sequestration in plasma membranes. It has been established that lysosomal sphingomyelinase is involved in cholesterol transport from lysosomes to the plasma membranes (52). Because SM avidly binds cholesterol (53), sphingomyelinase deficiency inhibits macrophage cholesterol efflux through promoting cholesterol sequestration by SM (52). Thus, it is possible that the reduced plasma membrane SM levels caused by Sptlc2 haploinsufficiency (Figure 2, C and D) might produce the inverse effect of sphingomyelinase deficiency with respect to macrophage cholesterol efflux. Sptlc2 haploinsufficiency would thus aid in cholesterol efflux by inducing less cholesterol sequestration in macrophage plasma membranes. It has been also shown that exogenously added SM significantly diminishes cholesterol efflux $(47,52)$ mediated by ABCA1 (47), which suggests that the increased SM content in the plasma membranes prevents cholesterol efflux.

In addition to SM, we found that levels of glycosphingolipids, including glucosylceramide and GM3, were decreased approximately 50\% in Sptlc2 $2^{+/-}$macrophage plasma membranes and lipid rafts (Figure 2, C and D). Glycosphingolipids have been implicated as potentially atherogenic lipids. Studies in $\mathrm{Apoe}^{-/-}$mice have indicated that exacerbated tissue glycosphingolipid accumulation resulting from $\alpha$-galactosidase deficiency promotes atherosclerosis (54), whereas decreased glycosphingolipids in mice treated with myriocin are associated with inhibited atherosclerosis (3436). However, direct inhibition of glycosphingolipid synthesis in Apoe ${ }^{-/-}$mice had no effect on atherosclerotic lesion development, although plasma glycosphingolipid concentrations were reduced $50 \%$ (55). In the present study, the reduced levels of glucosylceramide and GM3 in Sptlc2 $2^{+/-}$macrophages are not likely to have a major effect on atherogenesis, since we found that, in contrast to SM, exogenous supplementation of glucosylceramide or GM3

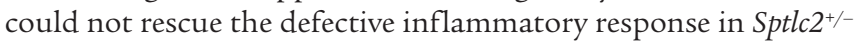
macrophages (Supplemental Figure 1, A and B). Therefore, it is unlikely that the reduction of glucosylceramide and GM3 in Sptlc2 $2^{+-}$macrophage plasma membranes and lipid rafts has any major effect on atherogenesis.
Another important intermediate in the sphingolipid de novo synthesis pathway is ceramide, one of the secondary messengers in cell signaling. We have previously reported that complete Sms 2 deficiency in macrophages significantly increases intracellular ceramide, but decreases SM levels, compared with WT controls (56). Moreover, Sms2 deficiency attenuates macrophage NF-кB activation (56) and promotes cholesterol efflux (57), thus decreasing atherosclerosis (57). In general, Sms2 deficiency and Sptlc2 haploinsufficiency yield similar phenotypes in terms of intracellular SM levels, NF-кB activation, and cholesterol efflux. However, in the present study, we did not observe significant changes of ceramide at the intracellular level or in plasma membranes or lipid rafts of Sptlc2 ${ }^{+/-}$macrophages (Figure 2, C and D, and Supplemental Table 1). Thus, ceramide levels may have a negligible role in mediating inflammation or cholesterol efflux, and therefore did not influence atherogenicity in our mouse models.

$\mathrm{S} 1 \mathrm{P}$, a biologically active lipid mediator, is another key byproduct of sphingolipid metabolism. It is present in plasma bound to HDL or albumin and in lower concentrations in tissues and has pleiotropic effects in different cell types, including macrophages, endothelial cells, and smooth muscle cells $(40,58,59)$. There are $5 \mathrm{G}$ protein-coupled receptors for S1P, S1PR1-S1PR5, in higher eukaryotes. These receptors are differentially expressed and play critical roles in multiple cellular functions involved in vascular physiology $(60,61)$. Monocytes/macrophages express mainly S1PR1 and S1PR2 (62). It has been reported that FTY-720, a synthetic S1P analog serving as an agonist for S1PR1 (but not S1PR2), inhibits atherosclerosis in $\mathrm{Ldlr}^{--}$and Apoe $e^{-/-}$mice $(63,64)$. S1PR2 deficiency leads to inhibition of macrophage proinflammatory activities and atherosclerosis in Apoe ${ }^{-/-}$mice (40). S1PR3 has also been found to promote macrophage recruitment in addition to S1PR2 (65). However, we did not find changes in intracellular S1P levels in Sptlc2 $2^{+-}$macrophages compared with controls (Supplemental Table 1). Plasma from Sptlc2 $2^{+--} \rightarrow L d l r^{--}$mice also showed no changes in S1P levels compared with controls (Supplemental Table 3). Moreover, we did not find significant changes of S1pr1 and S1pr2 mRNA in Sptlc2 ${ }^{+/-}$macrophages compared with controls (Supplemental Figure 8, A and B). These results suggest that neither S1P nor S1PR is involved in Sptlc2 haploinsufficiency-mediated reduction of inflammation.

It is known that macrophage cholesterol efflux and inflammation are inversely related to each other. Yvan-Charvet et al. reported that macrophage ABCA1 and ABCG1 deficiencies increase free cholesterol accumulation and increase cell signaling via TLRs (13). Zhu et al. reported that macrophage ABCA1 reduces MyD88dependent TLR trafficking to lipid rafts by reduction of lipid raft cholesterol (12). In addition, ABCA1 expression decreases cellular plasma membrane rigidity by reducing the formation of tightly packed lipid rafts (49). Therefore, more cholesterol efflux is related to less inflammation in macrophages. A recent report indicated that IL- 6 markedly induced ABCA1 expression and enhanced ABCA1-mediated cholesterol efflux from human macrophages to apoA-I (66). Our findings in this study clearly indicate that plasma membrane SM levels are also critical for the inverse relationship between macrophage cholesterol efflux and inflammation.

However, there exists a limitation in the mouse models used herein for atherosclerosis studies. Sptlc2 $2^{+-}$bone marrow also harbored Sptlc2 ${ }^{+/-}$immune cells - such as B cells, T cells, mast

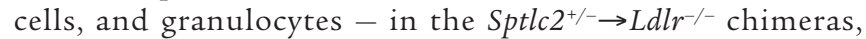
owing to the cells' hematopoietic origin. We cannot rule out 
the possible contributions of these important cells to atherogenesis. To address this issue, we transplanted bone marrow of Sptlc2-Flox/LysM-Cre mice into $\mathrm{Ldlr}^{-/-}$mice, and then evaluated atherosclerosis (Figure 9). LysM-Cre-mediated recombination resulted in deletion of the targeted gene in not only macrophages, but also the entire myeloid cell lineage (42). Therefore, we still cannot strictly rule out the contribution of granulocytes to atherogenesis, if any.

A key finding of this study was our demonstration of a novel mechanism of macrophage SPT-dependent regulation of lipid raft conformational and functional changes, which influenced inflammatory cell signaling and cholesterol homeostasis, affecting atherogenicity in mouse models. These findings suggest that macrophage SPT could be a potential target for therapeutic intervention against human atherosclerosis.

\section{Methods}

Mice and diets. The WT and heterozygous KO animals used in this study were all 8- to 10-week-old female mice, either littermates or with the same genetic background. All were fed a chow diet (Research Diets Inc.). Sptlc2 $2^{+/-}$ mice, originally on a 129 background, were backcrossed with C57BL/6 mice for 10 generations. $\mathrm{Ldlr}^{-/-}$mice (8-week-old females) and LysM-Cre transgenic mice were purchased from Jackson Laboratory. Sptlc2-Flox mice (41) and Sptlc2-Flox/LysM-Cre mice were prepared in house. Bone marrow transplantations were performed in lethally irradiated $\mathrm{Ldlr}^{/ /}$mice (8-weekold females), and after 8 weeks, all mice were switched to a Western-type diet $(0.15 \%$ cholesterol, $20 \%$ saturated fat) for 3-4 months.

Bone marrow-derived macrophage isolation. Mice were sacrificed, and femurs and tibiae were dissected out. Using needle and syringe, bone marrows were flushed directly into petri dishes containing DMEM plus $10 \%$ FBS and 20\% L-cell medium, then grown for 5-7 days. Differentiated macrophages attached to the bottom of the plates were harvested and used for all in vitro experiments.

Real-time PCR analysis for $m R N A$ quantification. Total RNA was isolated from treated or untreated WT and Sptlc2 $2^{+-}$bone marrow-derived macrophages after homogenizing in TRIzol reagent. Equal amounts of total RNA were used for cDNA preparation, using TaqMan RT Reagent (Applied Biosystems). Equal volumes of cDNA were also used for a real-time PCR reaction using $\mathrm{RT}^{2}$ Real Time SyBr Green/Rox PCR master mix (SA Biosciences), with specific primers for mRNA quantification and Gapdh as an internal control. Primer sequences were as follows: Sptlc1 forward, 5'-AGGGTTCTATGGCACATTTGATG-3'; Sptlc1 reverse, 5'-TGGCTTCTTCGGTCTTCATAAAC-3'; Sptlc2 forward, 5'-CAAAGAGCTTCGGTGCTTCAG-3'; Sptlc2 reverse, 5'-GAATGTGTGCGCAGGTAGTCTATC-3'; Sptlc3 forward, 5'-TGCAGCCAAGTATGATGAGTCTA-3'; Sptlc3 reverse, 5'-GCAGATGCACGATGGAAC-3'; Tnfa forward, 5'-CCCTCACACTCAGATCATCTTCT-3'; Tnfa reverse, 5'-GCTACGACGTGGGCTACAG-3'; Il6 forward, 5'-ACAACCACGGCCTTCCCTACTT-3', Il 6 reverse, 5'-CACGATTTCCCAGAGAACATGTG-3'; Gapdh forward, TGCACCACCAACTGCTTAGG, Gapdh reverse, GGATGCAGGGATGATGTTC.

SPT activity assay. Bone marrow-derived macrophages from WT and $S p t l 2^{+/-}$mice were harvested and homogenized. Macrophage homogenate (300 $\mu \mathrm{g}$ protein) from each sample were used as crude enzyme for the SPT reaction. SPT activity was measured with $\left[{ }^{14} \mathrm{C}\right]$-serine and palmitoyl-CoA as substrates, as previously described (37).

Lysenin treatment and cell mortality measurement. Bone marrow-derived macrophages were washed twice in PBS and incubated with 100 or $200 \mathrm{ng} /$ $\mathrm{ml}$ lysenin for 1 hour. Cell viability was measured using WST-1 cell proliferation reagent (Roche) according to the manufacturer's instructions.
Plasma membrane and lipid raft isolation from macrophages. Equal numbers of bone marrow-derived WT and Sptlc2 $2^{+-}$macrophages $\left(50 \times 10^{6}\right.$ cells $)$ were homogenized and used for plasma membrane isolation, using a kit from Biovision. They were checked for purity of preparation by Western blot, using cytoplasmic, total membrane, and plasma membrane fractions, with respective antibodies. Lipid rafts were isolated by a previously reported method (56).

Sphingolipid analyses by LC/MS/MS. Levels of SM, phosphatidylcholine, ceramide, and glycosphingolipids (glucosylceramide and GM3) were measured in WT and Sptlc2 $2^{+-}$bone marrow-derived macrophages by LC/MS/ MS, as previously described $(56,57)$.

Cell surface TLR 4 analysis by FACS. WT and Sptlc2+/- bone marrow-derived macrophages were treated overnight with $10 \mathrm{ng} / \mathrm{ml} \mathrm{LPS}$, washed, and harvested in PBS to make single-cell suspensions. Cells were then treated with Fc receptor block (mAb 2-4G2; BD Biosciences - Pharmingen) on ice for 1 hour and stained with primary antibodies to TLR4 (1:25 dilution; Abcam) for 1 hour on ice. After being washed, cells were stained with green fluorescent Alexa Fluor $488 \mathrm{~F}\left(\mathrm{ab}^{\prime}\right) 2$ fragment of goat anti-rabbit secondary antibodies (1:250 dilution; Invitrogen). After again being washed, cells were suspended in PBS containing $1 \mu \mathrm{g} / \mathrm{ml}$ propidium iodide (PI), then analyzed using FACScan with CellQuest (BD). Dead cells were excluded from the analysis by specific gating for PI staining.

In vitro macrophage Transwell migration assay. WT and Sptlc2 $2^{+-}$bone marrow-derived macrophages from $n=3$ mice were treated with $10 \mathrm{ng} / \mathrm{ml} \mathrm{LPS}$ for 16 hours, and the cells and culture media were harvested. Media were added to the lower chambers of 24-well Transwell plates (Corning), touching the bottom of the polycarbonate membrane inserts $(5-\mu \mathrm{m}$ pore size). Sptlc2 $2^{+-}$and WT macrophages $\left(2 \times 10^{5}\right.$ cells $)$ in DMEM were seeded on the corresponding top chambers and allowed to migrate for 4 hours at $37^{\circ} \mathrm{C}$ in cell culture incubator. After migration, media were aspirated, and the top of the membrane inserts were cleared of any cells using cotton swabs and checked under microscope. The bottom of the membranes with the migrated cells were washed with PBS, stained with DAPI, and observed under fluorescent microscope for counting.

Measurement of inflammatory cytokines TNF- $\alpha, I L-6$, and MCP-1. In vitro studies were done using WT and Sptlc2 $2^{+-}$bone marrow-derived macrophages from $n=3$ mice: for real-time PCR, mice were treated with $1 \mu \mathrm{g} / \mathrm{ml}$ LPS (Sigma-Aldrich) for 15 minutes and $500 \mu \mathrm{M}$ palmitate (Sigma-Aldrich) in $1 \%$ BSA for 30 minutes; for ELISA, mice were treated with $10 \mathrm{ng} / \mathrm{ml} \mathrm{LPS}$ and $50 \mu \mathrm{M}$ palmitate in $1 \% \mathrm{BSA}$ for 16 hours. The culture filtrate was separated and analyzed for TNF- $\alpha$, IL- 6 , and MCP-1 using ELISA kits (eBiosciences). In vivo studies were done using 10-week-old female WT and Sptlc2 ${ }^{+/-}$ mice $(n=9)$. Animals were retro-orbitally bled at 0 hours (for basal level) and 8 hours (after i.p. injection with $50 \mu \mathrm{g} / \mathrm{kg}$ body weight LPS). Plasma samples were collected and analyzed for TNF- $\alpha$, IL- 6 , and MCP-1 using ELISA kits (eBiosciences).

SM enrichment in macrophage plasma membranes. WT and Sptlc2 $2^{+-}$bone marrow-derived macrophages growing in plain DMEM were treated overnight with different concentrations of $\operatorname{SM}(0,10,25$, and $50 \mu \mathrm{M})$ from a 5-mM stock of SM dissolved in ethanol. Cells were washed with PBS 3 times, and a portion of the cells was subjected to lysenin treatment and assayed for mortality using WST-1 compound (Roche). The remaining cells were further treated with $10 \mathrm{ng} / \mathrm{ml}$ LPS overnight, after which the culture supernatant was collected and assayed for IL-6.

Western blot for macrophage ABCA1, ABCG1, and SR-B1. WT and Sptlc2+-- bone marrow-derived macrophages were lysed in $200 \mathrm{mM} \mathrm{NaCl}, 50 \mathrm{mM}$ Tris ( $\mathrm{pH}$ 7.5), $1 \mathrm{mM}$ EDTA, and $1 \%$ (v/v) protease inhibitor cocktail (Sigma-Aldrich). Cell debris was cleared by centrifugation at $8,200 \mathrm{~g}$ for 10 minutes. Lysates were subjected to SDS-PAGE and then transferred to nitrocellulose membranes. The blots were probed with antibodies against ABCA1 (Abcam), ABCG1 (Abcam), and SR-B1 (Novus). $\beta$-Actin was used as a loading control. 
Blots were developed by a chemiluminescence detection system (SuperSignal West detection kit; Pierce). The maximum intensity of each band was measured by Image-Pro Plus software (version 4.5; Media Cybernetics Inc.).

Cell surface ABCA1, ABCG1, and SR-B1 analysis by flow cytometry. WT and Sptlc2 $2^{+-}$bone marrow-derived macrophages were treated overnight with $50 \mu \mathrm{g} / \mathrm{ml}$ acetylated-LDL, washed, and harvested in PBS to make single-cell suspensions. Cells were then treated with $\mathrm{F}_{\mathrm{c}}$ receptor block (mAb 2-4G2; $\mathrm{BD}$ Biosciences - Pharmingen) on ice for 1 hour and stained with primary antibodies to ABCA1, ABCG1, or SR-B1 (all from Novus) at 1:50 dilution for 1 hour on ice. After being washed, cells were stained with green fluorescent Alexa Fluor 488-conjugated goat anti-rabbit secondary antibodies (1:100 dilution; Invitrogen). After again being washed, cells were suspended in PBS containing $1 \mu \mathrm{g} / \mathrm{ml}$ PI, then analyzed using FACScan with CellQuest (BD). Dead cells were excluded from the analysis by specific gating for PI staining.

Cholesterol efflux from macrophages ex vivo. WT and Sptlc2+- bone marrow-derived macrophages were labeled with $\left[{ }^{3} \mathrm{H}\right]$-cholesterol carried by acetylated-LDL. After labeling, cells were washed with PBS, equilibrated with DMEM plus $0.2 \%$ BSA for 1 hour, and incubated with $10 \mu \mathrm{g} / \mathrm{ml}$ purified human apoA-I or HDL in $0.5 \mathrm{ml}$ DMEM plus $0.2 \%$ BSA. Extracellular media were collected at 4 and 8 hours and centrifuged at $6,000 \mathrm{~g}$ for $10 \mathrm{~min}$ utes to remove residual cell debris and cholesterol crystals. Radioactivity in aliquots of supernatants was determined by liquid scintillation counting. The cells were finally lysed in $0.5 \mathrm{ml}$ of $0.1 \mathrm{M}$ sodium hydroxide plus $0.1 \%$ SDS, and the radioactivity in aliquots was determined. Cholesterol efflux was expressed as the percentage of the radioactivity released from the cells into the medium, relative to the total radioactivity in cells and medium.

Cholesterol efflux from macrophages in vivo. In vivo macrophage cholesterol efflux was measured by a method previously reported (39). Briefly, Sptlc2 ${ }^{+/-}$ or WT bone marrow-derived macrophages were loaded with cholesterol by incubation with acetylated-LDL and $\left[{ }^{3} \mathrm{H}\right]$-cholesterol. $2 \times 10^{6}$ labeled macrophages were injected i.p. into each C57BL/ 6 acceptor mouse. Plasma samples were collected at 12, 24, and 48 hours, and feces were collected at 24 and 48 hours; all were analyzed for tracer counts using liquid scintillation counter.

Bone marrow transplantation to replace PBMCs. Bone marrow cells were harvested from the tibiae of donor mice (Sptlc2 ${ }^{+/-}$and WT, or Sptlc2-Flox/ LysM-Cre and Sptlc2-Flox), as previously described. 18 total $\mathrm{Ldlr}^{-1}$-female mice (age 8 weeks) were lethally irradiated with $10 \mathrm{~Gy} .9$ of these animals were transplanted with Sptlc2 ${ }^{+/-}$or Sptlc2-Flox/LysM-Cre mouse bone marrow cells $\left(5 \times 10^{6}\right)$, and the other 9 with WT or Sptlc2-Flox mouse bone marrow, via the femoral vein, all within 3 hours of irradiation. We monitored the process of cell replacement by PCR, using genomic DNA from mouse PBMCs as templates.

Mouse atherosclerotic lesion measurement. Aortae were dissected, aortic arches were photographed, and aortic lesion en face assay was performed. For lesion analysis, sections were stained with Harris H\&E. Total intimal lesion area per cross section was quantified by taking the average of 10 sections spaced $20 \mu \mathrm{m}$ apart, beginning at the base of the aortic root. Histomorphological analysis of collagen was performed with Masson trichrome stain (Richard-Allan Scientific). Images were viewed and captured with a Nikon Labphoto 2 microscope equipped with a SPOT RT3 color video camera attached to a computerized imaging system with Image-Pro Plus software (version 4.5; Media Cybernetics Inc.).

Immunostaining of macrophage in the plaques. Sequential sections $10 \mu \mathrm{m}$ thick were stained with macrophage-specific antibody (MOMA). Primary antibodies were incubated for 1 hour at room temperature in 3\% serum matched to the species of the secondary antibodies. Biotinylated secondary antibodies were incubated for 30 minutes, followed by 45 minutes of horseradish peroxidase-conjugated streptavidin and visualization with diaminobenzidine. Nuclei were counterstained with hematoxylin. The mean area of staining per section per animal was determined using 10 sections for each animal. Staining areas were quantified with Image-Pro-Plus software.

Statistics. Each experiment was conducted at least 3 times. Unless otherwise indicated, data are expressed as mean \pm SD. Differences between 2 groups were analyzed by unpaired 2-tailed Student's $t$ test and among multiple groups by ANOVA followed by Student-Newman-Keuls test. A $P$ value less than 0.05 was considered significant.

Study approval. All experiments involving animals were approved by the Institutional Animal Care and Use Committee at SUNY Downstate Medical Center.

\section{Acknowledgments}

We thank Zhiqiang Li and Yan Li (SUNY Downstate Medical Center) for support and technical guidance; Wei Quan (SUNY Downstate Medical Center) for guidance using fluorescence microscopy; and Thomas Beyer, Hai H. Bui, David A. Peake, Youyan Zhang, and Phillip E. Sanders for help with LC/MS/MS analysis. C. Lou is supported by the China Scholarship Council (CSC; file no. 2011639024). This study was supported by NIH grant HL09341901A1 and VA Merit grant BX000900 to X.-C. Jiang.

Received for publication November 21, 2012, and accepted in revised form January 24, 2013.

Address correspondence to: Xian-Cheng Jiang, Department of Cell Biology, State University of New York, Downstate Medical Center, New York, New York, USA. Phone: 718.270.6701; Fax: 718.270.3732; E-mail: xjiang@downstate.edu.
1. Libby P. Inflammation in atherosclerosis. Nature. 2002;420(6917):868-874.

2. Mayo MW, Baldwin AS. The transcription factor NF-kappaB: control of oncogenesis and cancer therapy resistance. Biochim Biophys Acta. 2000;1470(2):M55-M62.

3. Karin M, Lin A. NF-kappaB at the crossroads of life and death. Nat Immunol. 2002;3(3):221-227.

4. Grilli M, Chiu JJ, Lenardo MJ. NF-kappa B and Rel: participants in a multiform transcriptional regulatory system. Int Rev Cytol. 1993;143:1-62.

5. Verma IM, Stevenson JK, Schwarz EM, Van Antwerp D, Miyamoto S. Rel/NF-kappa B/I kappa B family: intimate tales of association and dissociation. Genes Dev. 1995;9(22):2723-2735

6. Ross R. The pathogenesis of atherosclerosis: a perspective for the 1990s. Nature. 1993; 362(6423):801-809.

7. Freeman $\mathrm{M}$, et al. An ancient, highly conserved family of cysteine-rich protein domains revealed by cloning type I and type II murine macrophage scavenger receptors. Proc Natl Acad Sci U S A. 1990;87(22):8810-8814.

8. Cavelier C, Lorenzi I, Rohrer L, von Eckardstein A. Lipid efflux by the ATP-binding cassette transporters ABCA1 and ABCG1. Biochim Biophys Acta. 2006;1761(7):655-666

9. Jessup W, Gelissen IC, Gaus K, Kritharides L. Roles of ATP binding cassette transporters A1 and G1, scavenger receptor $\mathrm{BI}$ and membrane lipid domains in cholesterol export from macrophages. Curr Opin Lipidol. 2006;17(3):247-257.

10. Yvan-Charvet L, et al. Combined deficiency of ABCA1 and ABCG1 promotes foam cell accumulation and accelerates atherosclerosis in mice. J Clin Invest. 2007;117(12):3900-3908.

11. Out R, et al. Combined deletion of macrophage ABCA1 and ABCG1 leads to massive lipid accumulation in tissue macrophages and distinct atherosclerosis at relatively low plasma cholesterol levels.
Arterioscler Thromb Vasc Biol. 2008;28(2):258-264.

12. Zhu X, et al. Macrophage ABCA1 reduces MyD88dependent Toll-like receptor trafficking to lipid rafts by reduction of lipid raft cholesterol. J Lipid Res. 2010;51(11):3196-3206.

13. Yvan-Charvet $\mathrm{L}$, et al. Increased inflammatory gene expression in $\mathrm{ABC}$ transporter-deficient macrophages: free cholesterol accumulation, increased signaling via toll-like receptors, and neutrophil infiltration of atherosclerotic lesions. Circulation. 2008;118(18):1837-1847.

14. Simons K, Ikonen E. Functional rafts in cell membranes. Nature. 1997;387(6633):569-572.

15. Li Z, et al. Inhibition of sphingomyelin synthase (SMS) affects intracellular sphingomyelin accumulation and plasma membrane lipid organization. Biochim Biophys Acta. 2007;1771(9):1186-1194.

16. Shaul PW, Anderson RG. Role of plasmalemmal caveolae in signal transduction. Am J Physiol. 1998;275(5 pt 1):L843-851. 
17. Futerman AH, Hannun YA. The complex life of simple sphingolipids. EMBO Rep. 2004;5(8):777-782.

18. Holthuis JC, van Meer G, Huitema K. Lipid microdomains, lipid translocation and the organization of intracellular membrane transport (Review). $\mathrm{Mol}$ Membr Biol. 2003;20(3):231-241.

19. Kim S, Watarai M, Suzuki H, Makino S, Kodama $\mathrm{T}$, Shirahata T. Lipid raft microdomains mediate class A scavenger receptor-dependent infection of Brucella abortus. Microb Pathog. 2004;37(1):11-19.

20. Graf GA, Connell PM, van der Westhuyzen DR, Smart EJ. The class B, type I scavenger receptor promotes the selective uptake of high density lipoprotein cholesterol ethers into caveolae.J Biol Chem. 1999;274(17):12043-12048

21. Rhainds D, Bourgeois P, Bourret G, Huard K, Falstrault $\mathrm{L}$, Brissette $\mathrm{L}$. Localization and regulation of SR-BI in membrane rafts of HepG2 cells. J Cell Sci. 2004;117(pt 15):3095-3105.

22. von Arnim CA, et al. The low density lipoprotein receptor-related protein (LRP) is a novel beta-secretase (BACE1) substrate. J Biol Chem. 2005;280(18):17777-17785.

23. Zeng Y, Tao N, Chung KN, Heuser JE, Lublin DM. Endocytosis of oxidized low density lipoprotein through scavenger receptor CD36 utilizes a lipid raft pathway that does not require caveolin-1.J Biol Chem. 2003;278(46):45931-45936.

24. Lee HK, Dunzendorfer S, Soldau K, Tobias PS. Double-stranded RNA-mediated TLR3 activation is enhanced by CD14. Immunity. 2006;24(2):153-163.

25. Wang R, Town T, Gokarn V, Flavell RA, Chandawarkar RY. HSP70 enhances macrophage phagocytosis by interaction with lipid raft-associated TLR-7 and upregulating P38 MAPK and PI3K pathways. J Surg Res. 2006;136(1):58-69.

26. Nakahira K, et al. Carbon monoxide differentially inhibits TLR signaling pathways by regulating ROS-induced trafficking of TLRs to lipid rafts. J Exp Med. 2006;203(10):2377-2389.

27. Szabo G, Dolganiuc A, Dai Q, Pruett SB. TLR4, ethanol, and lipid rafts: a new mechanism of ethanol action with implications for other receptor-mediated effects. J Immunol. 2007;178(3):1243-1249.

28. Merrill AH. Characterization of serine palmitoyltransferase activity in Chinese hamster overy cells. Biochim Biophys Acta. 1983;754(3):284-291.

29. Weiss B, Stoffel W. Human and murine serine-palmitoyl-CoA transferase - cloning, expression and characterization of the key enzyme in sphingolipid synthesis. Eur J Biochem. 1997;249(1):239-247.

30. Hanada K, Hara T, Nishijima M. Purification of the serine palmitoyltransferase complex responsible for sphingoid base synthesis by using affinity peptide chromatography techniques. J Biol Chem. 2000;275(12):8409-8415.

31. Hornemann T, Richard S, Rutti MF, Wei Y, von Eckardstein A. Cloning and initial characterization of a new subunit for mammalian serine-palmitoyltransferase. J Biol Chem. 2006;281(49):37275-37281.

32. Hornemann T, Wei Y, von Eckardstein A. Is the mammalian serine palmitoyltransferase a high-molecular-mass complex? Biochem J. 2007;405(1):157-164

33. Han G, et al. Identification of small subunits of mammalian serine palmitoyltransferase that con- fer distinct acyl-CoA substrate specificities. Proc Natl Acad Sci U S A. 2009;106(20):8186-8191.

34. Park TS, et al. Inhibition of sphingomyelin synthesis reduces atherogenesis in apolipoprotein E-knockout mice. Circulation. 2004;110(22):3465-3471.

35. Hojjati MR, et al. Effect of myriocin on plasma sphingolipid metabolism and atherosclerosis in apoE-deficient mice. $J$ Biol Chem. 2005;280(11):10284-10289.

36. Glaros EN, et al. Inhibition of atherosclerosis by the serine palmitoyl transferase inhibitor myriocin is associated with reduced plasma glycosphingolipid concentration. Biochem Pharmacol. 2007;73(9):1340-1346.

37. Hojjati MR, Li Z, Jiang XC. Serine palmitoyl-CoA transferase (SPT) deficiency and sphingolipid levels in mice. Biochim Biophys Acta. 2005;1737(1):44-51.

38. Rao KM. MAP kinase activation in macrophages. J Lenkoc Biol. 2001;69(1):3-10.

39. Zhang Y, Zanotti I, Reilly MP, Glick JM, Rothblat GH, Rader DJ. Overexpression of apolipoprotein A-I promotes reverse transport of cholesterol from macrophages to feces in vivo. Circulation. 2003;108(6):661-663

40. Wang F, et al. Sphingosine-1-phosphate receptor-2 deficiency leads to inhibition of macrophage proinflammatory activities and atherosclerosis in apoEdeficient mice. J Clin Invest. 2010;120(11):3979-3995.

41. Li Z, et al. Liver-specific deficiency of serine palmitoyltransferase subunit 2 decreases plasma sphingomyelin and increases apolipoprotein E levels. J Biol Chem. 2009;284(39):27010-27019.

42. Clausen BE, Burkhardt C, Reith W, Renkawitz $\mathrm{R}$, Forster I. Conditional gene targeting in macrophages and granulocytes using LysMcre mice. Transgenic Res. 1999;8(4):265-277.

43. Mitsutake S, et al. Dynamic modification of sphingomyelin in lipid microdomains controls development of obesity, fatty liver, and type 2 diabetes. J Biol Chem. 2011;286(32):28544-28555.

44. Li Z, et al. Reducing plasma membrane sphingomyelin increases insulin sensitivity. Mol Cell Biol. 2011;31(20):4205-4218

45. Miyaji M, et al. Role of membrane sphingomyelin and ceramide in platform formation for Fas-mediated apoptosis. J Exp Med. 2005;202(2):249-259.

46. Huang ZH, Gu D, Lange Y, Mazzone T. Expression of scavenger receptor BI facilitates sterol movement between the plasma membrane and the endoplasmic reticulum in macrophages. Biochemistry. 2003;42(13):3949-3955.

47. Nagao K, Takahashi K, Hanada K, Kioka N, Matsuo M, Ueda K. Enhanced apoA-I-dependent cholesterol efflux by ABCA1 from sphingomyelindeficient Chinese hamster ovary cells. J Biol Chem. 2007;282(20):14868-14874.

48. Fessler MB, Parks JS. Intracellular lipid flux and membrane microdomains as organizing principles in inflammatory cell signaling. J Immunol. 2011;187(4):1529-1535

49. Landry YD, Denis M, Nandi S, Bell S, Vaughan AM, Zha X. ATP-binding cassette transporter A1 expression disrupts raft membrane microdomains through its ATPase-related functions. J Biol Chem. 2006;281(47):36091-36101.

50. Peshavariya H, Dusting GJ, Di Bartolo B, Rye KA,
Barter PJ, Jiang F. Reconstituted high-density lipoprotein suppresses leukocyte NADPH oxidase activation by disrupting lipid rafts. Free Radic Res. 2009;43(8):772-782.

51. Fielding PE, Russel JS, Spencer TA, Hakamata H, Nagao K, Fielding CJ. Sterol efflux to apolipoprotein A-I originates from caveolinrich microdomains and potentiates PDGFdependent protein kinase activity. Biochemistry. 2002;41(15):4929-4937.

52. Leventhal AR, Chen W, Tall AR, Tabas I. Acid sphingomyelinase-deficient macrophages have defective cholesterol trafficking and efflux. J Biol Chem. 2001;276(48):44976-44983.

53. Slotte JP. Sphingomyelin-cholesterol interactions in biological and model membranes. Chem Phys Lipids. 1999;102(1-2):13-27.

54. Bodary PF, et al. Alpha-galactosidase A deficiency accelerates atherosclerosis in mice with apolipoprotein E deficiency. Circulation. 2005;111(5):629-632.

55. Glaros EN, Kim WS, Rye KA, Shayman JA, Garner B. Reduction of plasma glycosphingolipid levels has no impact on atherosclerosis in apolipoprotein E-null mice. J Lipid Res. 2008;49(8):1677-1681.

56. Hailemariam TK, et al. Sphingomyelin synthase 2 deficiency attenuates NFkappaB activation. Arterioscler Thromb Vasc Biol. 2008;28(8):1519-1526.

57. Liu JY, Lo PC, Jiang XJ, Fong WP, Ng DK. Synthesis and in vitro photodynamic activities of di-alphasubstituted zinc(ii) phthalocyanine derivatives. Dalton Trans. 2009;(21):4129-4135.

58. Brinkmann V, Cyster JG, Hla T. FTY720: sphingosine 1-phosphate receptor-1 in the control of lymphocyte egress and endothelial barrier function. Am J Transplant. 2004;4(7):1019-1025.

59. Kluk MJ, Hla T. Role of the sphingosine 1-phosphate receptor EDG-1 in vascular smooth muscle cell proliferation and migration. Circ Res. 2001;89(6):496-502.

60. Sanchez T, Hla T. Structural and functional characteristics of S1P receptors. J Cell Biochem. 2004;92(5):913-922.

61. Spiegel S, Milstien S. Sphingosine-1-phosphate: an enigmatic signalling lipid. Nat Rev Mol Cell Biol. 2003;4(5):397-407.

62. Hughes JE, Srinivasan S, Lynch KR, Proia RL, Ferdek P, Hedrick CC. Sphingosine-1-phosphate induces an antiinflammatory phenotype in macrophages. Circ Res. 2008;102(8):950-958.

63. Nofer JR, et al. FTY720, a synthetic sphingosine 1 phosphate analogue, inhibits development of atherosclerosis in low-density lipoprotein receptordeficient mice. Circulation. 2007;115(4):501-508.

64. Keul P, et al. The sphingosine-1-phosphate analogue FTY720 reduces atherosclerosis in apolipoprotein E-deficient mice. Arterioscler Thromb Vasc Biol. 2007;27(3):607-613.

65. Keul P, et al. Sphingosine-1-phosphate receptor 3 promotes recruitment of monocyte/macrophages in inflammation and atherosclerosis. Circ Res. 2011;108(3):314-323.

66. Frisdal E, et al. Interleukin-6 protects human macrophages from cellular cholesterol accumulation and attenuates the pro-inflammatory response. J Biol Chem. 2011;286(35):30926-30936. 